\title{
On the impact of damping on the dispersion curves of a locally resonant metamaterial: modelling and experimental validation
}

\author{
Lucas Van Belle ${ }^{\mathrm{a}, \mathrm{b}, *}$, Claus Claeys ${ }^{\mathrm{a}, \mathrm{b}}$, Elke Deckers ${ }^{\mathrm{a}, \mathrm{b}}$, Wim Desmet ${ }^{\mathrm{a}, \mathrm{b}}$ \\ ${ }^{a}$ KU Leuven, Department of Mechanical Engineering, Division PMA, Celestijnenlaan 300 - box 2420, 3001 Heverlee, Belgium \\ ${ }^{b}$ Member of Flanders Make
}

\begin{abstract}
Recently, locally resonant metamaterials have come to the fore in noise and vibration control engineering, showing great potential due to their superior noise and vibration attenuation performance in targeted and tunable frequency ranges, referred to as stop bands. Damping has an important influence on the performance of these materials, broadening the frequency range of attenuation at the expense of peak attenuation. As a result, understanding and including the effects of damping is necessary to more accurately predict the attenuation performance of these locally resonant metamaterials. Classically, these often periodic structures are analysed using a unit cell modelling approach to predict wave propagation and thus stop band behaviour, discarding damping. In this work, a unit cell method including damping is used to analyse the complex dispersion curves of a locally resonant metamaterial design. The wave solutions in and around the stop band frequency range are compared to those of the unit cell method discarding damping. The influence on the dispersion curves of damping in resonator and host structure is discussed and the obtained dispersion curves are validated through an experimental dispersion curve measurement based on an Extended Inhomogeneous Wave Correlation method using Scanning Laser Doppler Vibrometry for a metamaterial plate manufactured at a representative scale. Excellent agreement is obtained between the numerically predicted and experimentally retrieved dispersion curves.
\end{abstract}

Keywords: Locally resonant metamaterial, Stop band, Unit cell analysis, Damping, Dispersion curves, Inhomogeneous Wave Correlation

\section{Introduction}

Over the past decades, tightening ecologic as well as economic requirements have given rise to the need for lightweight materials and designs [1]. Because of their increased stiffness over mass ratio, lightweight materials exhibit worse noise, vibration and harshness (NVH) behaviour. This conflicts with customer expectations and the increasingly stringent legal regulations regarding NVH exposure [2] for which current countermeasures are typically bulky or heavy. Novel low mass and compact volume NVH solutions are required to face the challenging task of merging both favourable NVH suppression performance and lightweight requirements.

*Corresponding author, Tel.: +3216322041, http://www.mech.kuleuven.be/, e-mail address: lucas.vanbelle@kuleuven.be. 
Among these novel NVH solutions, in recent years, innovative materials relying on stop band behaviour have been receiving increasingly more attention in noise and vibration engineering. Stop bands are frequency ranges in which no free wave propagation is possible [3], which allow these materials to exhibit superior NVH suppression performance. These stop band materials are generally subdivided into phononic crystals (PCs) and acoustic or locally resonant metamaterials (LRMs), for which the formation of stop bands relies on different mechanisms [4, 5]. PCs are structured materials with periodic variations in properties. Their stop band behaviour relies on Bragg scattering, causing frequency zones of destructive interference between reflected and transmitted waves, linked to the length scale of periodicity $[5,6,7,8]$. The formation of stop bands in LRMs does not rely on periodicity or Bragg scattering, but emerges from the addition of local resonances to a host structure on a subwavelength scale, a spatial scale much smaller than the wavelength to be affected $[5,9,10,11]$. Stop band behaviour in LRMs is known to emerge from the Fano-type interference between incoming travelling waves and the waves re-radiated by these resonators around their resonance frequency $[12,13]$. Since LRMs do not require periodicity and are of subwavelength nature, they are more suitable to create targeted and tunable stop bands and thus superior NVH attenuation in the hard-to-address lower frequency range [13]. Therefore, in this work, LRMs are considered for further investigation.

LRMs have been widely investigated and their stop band effect has been established experimentally for a variety of realisations, ranging from one-dimensional beam-like structures to three-dimensional solid structures $[6,10,14,15,16,17,18,19,20]$. The analysis of LRMs, in order to numerically predict the attenuation performance, classically discards the presence of damping as the stop band mechanism causing attenuation does not rely on damping. However, as the materials used are often plastic or rubber-like, measured attenuation performances are often clearly affected by the presence of damping in these LRM constituents and are showing discrepancies with the numerical attenuation predictions. Only a limited number of works investigate the impact of damping on the LRM attenuation performance, often confined to mass-spring-damper lattices/ resonant structures [13, 21, 22, 23, 24]. Effects of damping on more complex and practically realisable LRM structures are considered in [25, 26, 27], however, experimental validation is lacking. The main findings in these studies are in general that the presence of damping broadens the frequency range of attenuation with respect to the original stop band, at the cost of a decreased peak attenuation performance inside the original stop band. This work aims at (i) incorporating and analysing the presence of damping in the constituents (host structure, resonant structure and both combined) of a realisable two-dimensional plate type LRM design for the numerical attenuation performance prediction and (ii) validating the results with experimental measurements. By gaining insight in these effects, the presence of damping can then be exploited in future metamaterial designs to e.g. combine multiple stop band regions $[20,28]$ or add specific damping treatments to or in between constituents [29], in order for these LRMs to be applicable for NVH suppression in a broader frequency range, while relying on trustworthy attenuation performance predictions.

The attenuation performance of stop band materials, in particular LRMs, is generally assessed by means of dispersion curve analysis, describing the wave propagation behaviour in the structure by representing the relation 
between the angular frequency $\omega$ or frequency $f$ and the wavenumber $k$ or propagation constant $\mu$. To this end, the often, yet not strictly required, periodic nature of these metamaterials is exploited. Making use of infinite periodic structure theory and the Bloch-Floquet theorem allows the metamaterial structure to be represented by a single unit cell (UC), which results in an eigenvalue problem (EVP) in $\omega$ and $k$ or $\mu$. The solution of this EVP yields dispersion curves from which the wave propagation and thus stop band behaviour can be predicted $[3,30]$. The dispersion EVP is generally solved either for imposed freely propagating waves, thus real wavenumbers $k$ or propagation constants $\mu$, referred to as the "inverse", $\omega(k)$ or $\omega(\mu)$ method, or for imposed time harmonic motion, thus real frequencies $\omega$, referred to as the "direct", $k(\omega)$ or $\mu(\omega)$ method [30, 31, 32]. In what follows, these methods will be referred to as the $\omega(\mu)$ and $\mu(\omega)$ approaches.

If no damping is considered in the UC, both $\omega(\mu)$ or $\mu(\omega)$ can be applied. Real, imaginary and complex wave solutions can be obtained, indicating freely propagating, evanescent and decaying waves respectively, in time or spatial domain. Classically, only the $\omega(\mu)$ method is applied and the dispersion curves are calculated for freely propagating, thus real $\mu$ along the edges of the irreducible Brillouin zone (IBZ) in the wave domain, referred to as the irreducible Brillouin contour (IBC) $[3,33]$. Stop bands emerge as frequency zones in which no freely propagating wave solutions are obtained for the dispersion curves along the entire IBC. If damping is considered in the UC model, the solutions of the EVP become complex and the $\omega(\mu)$ or $\mu(\omega)$ approach can be used to analyse the complex dispersion curves [31]. For the $\omega(\mu)$ approach, imposing freely propagating waves, thus real propagation constants $\mu$, leads to complex frequency solutions $\omega$ corresponding to temporally decaying waves. The $\omega(\mu)$ approach has been developed and applied to analyse the effects of damping on dispersion relations for PCs in [32, 34, 35] and for LRMs in [21, 36]. The $\mu(\omega)$ approach on the other hand imposes time harmonic wave motion, thus real frequencies $\omega$, and solves the EVP for complex propagation constants $\mu$, corresponding to spatially decaying waves. The effects of damping on the complex $\mu$ dispersion curves are analyzed for PCs in $[32,37,38]$ and for LRMs $[25,27]$. In what follows, the main findings for each approach in the presence of damping are summarized.

Using the $\omega(\mu)$ approach, Hussein et al. [34] point out that, for a two-dimensional PC with Rayleigh damping, increasing damping shifts the dispersion curves down in frequency, while the band gap size can drop abruptly due to branch overtaking. For one-dimensional lumped mass-spring PCs, Hussein and Frazier [35] observe general viscous damping to cause branch overtaking and branch cut-off phenomena, which can result in sudden changes in the band gap size and formation of partial wavenumber band gaps. For two-dimensional PCs with proportional and general viscous damping, the dispersion curves are observed to shift down in frequency with damping. In [21] Hussein and Frazier report a metadamping phenomenon in one-dimensional viscously damped mass-in-mass chain LRMs, leading to considerable amplification of the dissipation compared to their PC counterparts. In [36], they further investigate viscous and viscoelastic damping effects in one-dimensional PCs and LRMs, observing a narrowing of the band gaps for viscous damping, with an inverse behaviour for viscoelastic damping. Andreassen and Jensen [32] report an upward frequency shift of the dispersion curves when structural damping is increased in a two-dimensional PC.

The $\mu(\omega)$ approach is used by Farzbod and Leamy [37] to analyse generally damped one- and two-dimensional 
mass-spring PCs. Viscous damping is found to cause wavenumber band gaps for the real part of the wavenumber due the dispersion curves bending back towards the origin, while causing a reduction of the imaginary part of the wavenumber inside the band gap, which helps the wave to propagate. They report that considering only the IBC, as is typically done using the $\omega(\mu)$ approach, does not capture all extreme values of $\omega$ in presence of damping. Andreassen and Jensen [32] issue a similar remark in their analysis of two-dimensional PCs with structural damping. Additional evanescent modes in the band gap region are found inside the IBZ, which are not found along the IBC. Moiseyenko and Laude [38] find material losses in two-dimensional PCs to cause a pronounced rounding effect of the dispersion curves at zero group velocity points, including the edges of the IBZ, while the real part of $\mu$ is observed to be more affected than the imaginary part. A realisable two-dimensional periodically stubbed LRM plate is considered by Collet et al. [25], for which the stop band is observed to be smoothed out due to the presence of material damping. Recently, a more thorough analysis of the visco-elastic effects in three-phase LRMs is conducted in [27] using the $\mu(\omega)$ approach for a single propagation direction. Damping in the hosting matrix is found to decrease the LRM attenuation performance, while damping in the resonator coating improves the attenuation bandwidth at the cost of reduced peak attenuation.

Both the $\omega(\mu)$ and $\mu(\omega)$ approaches for the calculation of dispersion curves have been widely investigated. These methods have been compared by Mace and Manconi in [39] for viscoelamiated structures, showing good agreement for the retrieved loss factors. In [32], however, good agreement between both methods is found for PCs with damping only for low levels of material dissipation and long wavelengths. It is also highlighted by Mace in [40] that these two approaches are only a subset of possible solutions to the dispersion EVP in $\omega$ and $\mu$. Recently, Frazier and Hussein [24] have proposed an iterative algorithm, where simultaneously complex $\omega$ and $\mu$ dispersion curves are obtained for one-dimensional viscously damped spring-mass LRMs. The closing of the band gaps with damping, causing a broadening of the frequency range of attenuation at a reduced peak performance, is observed in conjunction with band gap shifts, branch cut-off and branch overtaking phenomena, combining the observed effects of both $\mu(\omega)$ and $\omega(\mu)$ approaches.

From the previous overview it is clear that, while the influence of damping on the dispersion curves for PCs has already been part of extensive research, the analysis of damping in LRMs is less prominent and mainly limited to academic mass-spring-damper chains, lattices or systems. In general, the presence of damping is found to broaden the frequency range of attenuation with respect to the original stop band, at the cost of a decreased peak attenuation performance. To develop a deeper understanding of the influence of damping on the time harmonic stop band behaviour of LRMs, in this work, the $\mu(\omega)$ approach is applied to predict and validate experimentally the attenuation performance for a general UC model including damping, based on the Wave and Finite Element Method (WFEM) approach [41]. As current literature is mainly limited to academic examples, in this work, the method is applied to a realisable plate type LRM consisting of resonant structures attached to an aluminium plate host structure, containing experimentally retrieved damping values. To address the issue of possible insufficiency of the IBC [32,37], the influence of damping on the attenuation performance is investigated by considering complex $\mu$ dispersion curves for various propagation 
directions throughout the IBZ. The results are analysed in the complex $\mu$ space to analyse the effect of damping in the different metamaterial constituents on the attenuation performance.

In current literature, the assessment of the impact of damping on the dispersion curves using either $\mu(\omega)$ or $\omega(\mu)$ approach is also limited to numerical analyses. In this work, the numerically obtained attenuation predictions are therefore validated experimentally by means of dispersion curve measurements on a manufactured LRM plate of representative dimensions. Since damping is considered, a method should be used that allows retrieving dispersion curves in damped two-dimensional structures. In [42] Roozen et al. measure dispersion curves for poro-visco-elastic materials, by applying the Fourier transform to Scanning Laser Doppler Vibrometer (SLDV) measurements of the out-of-plane velocity response along a straight line of the structure subjected to shaker excitation. Airoldi et al. [15] measure dispersion curves of a tunable metamaterial beam equipped with a periodic array of resonant shunted piezoelectric patches, by applying the Fourier transform to the out-of-plane displacement response of the beam excited with a pulse generated by a piezoelectric actuator. In [43], a similar approach is followed by considering the response along a waveguide path realised by a periodic array of piezoelectric patches on a two-dimensional PC. In [44], Berthaut et al. present a wavenumber space analysis method for the measurement of the flexural wavenumber of damped two-dimensional structures for various propagation directions, named the Inhomogeneous Wave Correlation (IWC) method. In this work, the IWC method is extended with the experimental excitation location, the Extended IWC (EIWC) method, in order to obtain a more accurate damped dispersion curve measurement. Experimental dispersion curves for various propagation directions are retrieved from SLDV displacement field measurements of a manufactured metamaterial plate and compared to the numerically predicted damped dispersion curves.

This paper is organised as follows. Section 2 presents the UC modelling procedure for general UC models including damping. In Sec. 3 the LRM design is introduced. Next, the numerical dispersion curve predictions obtained with the introduced UC modelling procedure are discussed and the effect of damping in resonator and host structure is discussed. In Sec. 4 the EIWC method is explained and the measurement setup is described. The measured dispersion curves are presented and compared to the numerical predictions obtained using the UC modelling procedure. Section 5 summarises the main findings.

\section{Unit cell modelling with damping}

In this section, the unit cell modelling procedure is explained. LRMs, although often periodic, do not strictly require periodicity to obtain resonant stop band behaviour. Assuming periodicity, however, allows their modelling to be performed through a UC analysis, using the Bloch-Floquet theorem. To this end, a model of a single representative UC is required, in combination with Bloch-Floquet periodicity boundary conditions. In this work, two-dimensional planar periodicity is considered for a rectangular UC, while the UC is modelled using the Finite Element Method 
(FEM), based on the WFEM method [41], which allows complex structures to be modelled and readily available FEM packages to be used.

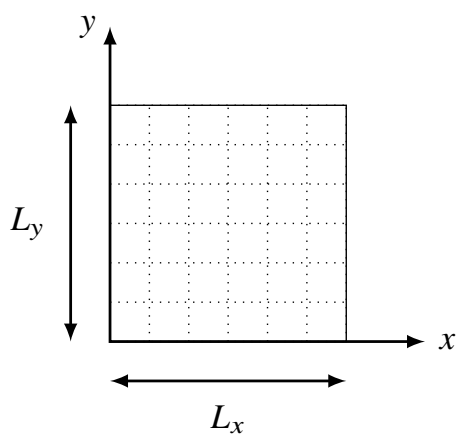

(a)

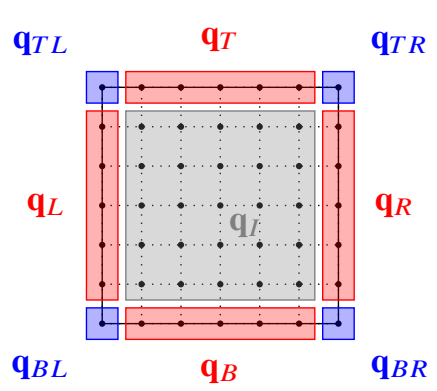

(b)

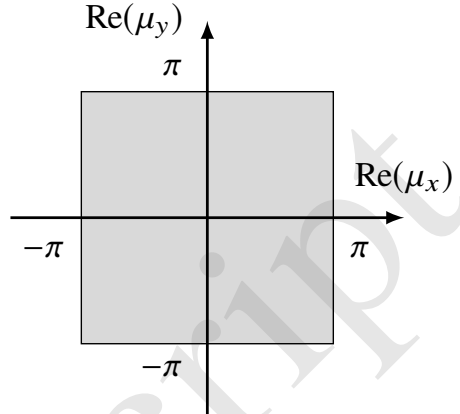

(c)

Figure 1 General rectangular unit cell of a 2D periodic structure. (a) UC dimensions, (b) FE model node groups and (c) first Brillouin zone.

A general, rectangular UC of dimensions $L_{x} \times L_{y}$ is shown in Fig. 1a. Considering time harmonic motion, the FE equations of motion for the UC containing $N$ degrees of freedom (DOFs) are in general given as:

$$
\hat{\mathbf{D}}(\omega) \hat{\mathbf{q}}=\hat{\mathbf{f}}
$$

with $(N \times 1)$ generalised displacement and force vectors $\hat{\mathbf{q}}$ and $\hat{\mathbf{f}}$ and the frequency dependent $(N \times N)$ dynamic stiffness matrix $\hat{\mathbf{D}}(\omega)$, which is in general complex in the presence of damping. The generalised displacement vector $\hat{\mathbf{q}}$ collects all the UC DOFs and is partitioned according to the DOFs on the boundary and on the interior of the UC, as shown in Fig. 1b:

$$
\hat{\mathbf{q}}=\left[\mathbf{q}_{B L}^{T} \mathbf{q}_{B R}^{T} \mathbf{q}_{T L}^{T} \mathbf{q}_{T R}^{T} \mathbf{q}_{L}^{T} \mathbf{q}_{R}^{T} \mathbf{q}_{B}^{T} \mathbf{q}_{T}^{T} \mathbf{q}_{I}^{T}\right]^{T}
$$

The same partitioning is applied to $\hat{\mathbf{f}}$ and to $\hat{\mathbf{D}}_{i, j}$ with $i, j \in\{B L, B R, T L, T R, L, R, B, T, I\}$.

The Bloch-Floquet theorem states that, for plane wave propagation through the two-dimensional periodic structure, the generalised displacements and forces at the boundaries of a rectangular UC are scaled with factors $\lambda_{x}=e^{\mathrm{i} \mu_{x}}$ and $\lambda_{y}=e^{\mathrm{i} \mu_{y}}$, in which $\mathrm{i}^{2}=-1$, when moving from one UC to the next along the direct lattice vectors $\boldsymbol{d}_{x}$ and $\boldsymbol{d}_{y}$ of UC lengths $L_{x}$ and $L_{y}$ respectively. The complex propagation constants $\mu_{i}$ along a lattice vector $\boldsymbol{d}_{i}$ are defined as:

$$
\mu_{i}=k \cdot d_{i}
$$

with $k$ the wavevector, and $\operatorname{Re}\left(\mu_{i}\right)$ and $\operatorname{Im}\left(\mu_{i}\right)$ representing wave propagation and decay, respectively. Due to periodicity, all the propagation constant information for the infinite periodic structure is contained in the first Brillouin zone (Fig. $1 \mathrm{c})$, with $\mu_{x} \in[-\pi, \pi]$ and $\mu_{y} \in[-\pi, \pi][3,30]$. 
Applying Bloch-Floquet periodicity conditions to the UC boundaries, the displacement continuity is written in matrix form as follows, in terms of the boundary DOFs $\mathbf{q}=\hat{\mathbf{q}} \backslash \mathbf{q}_{I}[41]$ :

$$
\mathbf{q}=\boldsymbol{\Lambda}_{R}\left\{\begin{array}{c}
\mathbf{q}_{B L} \\
\mathbf{q}_{L} \\
\mathbf{q}_{B}
\end{array}\right\} ; \quad \boldsymbol{\Lambda}_{R}=\left[\begin{array}{ccc}
\mathbf{Q}_{B L, B L} & \mathbf{0} & \mathbf{0} \\
\lambda_{x} \mathbf{Q}_{B R, B L} & \mathbf{0} & \mathbf{0} \\
\lambda_{y} \mathbf{Q}_{T L, B L} & \mathbf{0} & \mathbf{0} \\
\lambda_{x} \lambda_{y} \mathbf{Q}_{T R, B L} & \mathbf{0} & \mathbf{0} \\
\mathbf{0} & \mathbf{Q}_{L, L} & \mathbf{0} \\
\mathbf{0} & \lambda_{x} \mathbf{Q}_{R, L} & \mathbf{0} \\
\mathbf{0} & \mathbf{0} & \mathbf{Q}_{B, B} \\
\mathbf{0} & \mathbf{0} & \lambda_{y} \mathbf{Q}_{T, B}
\end{array}\right]
$$

and force equilibrium, with $\mathbf{f}$ the vector of generalised boundary forces, is given in matrix form by:

$$
\begin{aligned}
\boldsymbol{\Lambda}_{L} \mathbf{f} & =\mathbf{0} \\
\boldsymbol{\Lambda}_{L} & =\left[\begin{array}{cccccccc}
\mathbf{Q}_{B L, B L} & \lambda_{x}^{-1} \mathbf{Q}_{B L, B R} & \lambda_{y}^{-1} \mathbf{Q}_{B L, T L} & \lambda_{x}^{-1} \lambda_{y}^{-1} \mathbf{Q}_{B L, T R} & \mathbf{0} & \mathbf{0} & \mathbf{0} & \mathbf{0} \\
\mathbf{0} & \mathbf{0} & \mathbf{0} & \mathbf{0} & \mathbf{Q}_{L, L} & \lambda_{x}^{-1} \mathbf{Q}_{L, R} & \mathbf{0} & \mathbf{0} \\
\mathbf{0} & \mathbf{0} & \mathbf{0} & \mathbf{0} & \mathbf{0} & \mathbf{0} & \mathbf{Q}_{B, B} & \lambda_{y}^{-1} \mathbf{Q}_{B, T}
\end{array}\right]
\end{aligned}
$$

The UC boundary nodes should be located at the corresponding positions on either side of the UC for the BlochFloquet boundary conditions to be applicable. In above Eq. (4) and (5), the matrices $\mathbf{Q}_{i, j}$ are selection matrices for the corresponding DOFs between each of the UC DOF groups with $\mathbf{Q}_{j, i}=\mathbf{Q}_{i, j}^{T}$ and $\mathbf{Q}_{i, i}$ being unit matrices $\mathbf{I}_{i, i}$. These are introduced, as the amount of corresponding nodes in each of the boundary node groups may differ. The UC orientation is chosen such that $\mathbf{q}_{B L}$ contains the corresponding nodes for each of the other corner groups $\mathbf{q}_{B R}, \mathbf{q}_{T L}$ and $\mathbf{q}_{T R}$. It is noted that boundary nodes, which are not involved in the periodicity boundary conditions, because of lack of corresponding nodes on the UC boundaries, can be assigned to $\mathbf{q}_{I}$.

Since the interior DOFs $\mathbf{q}_{I}$ are not involved in the periodicity boundary conditions, the problem can be reduced to the boundary nodes $\mathbf{q}$ by dynamic condensation. Following [41], if no external forces are acting on the internal nodes, $\mathbf{f}_{I}=\mathbf{0}$, Eq. (1) can be rewritten as:

$$
\hat{\mathbf{D}}\left\{\begin{array}{c}
\mathbf{q} \\
\mathbf{q}_{I}
\end{array}\right\}=\left\{\begin{array}{l}
\mathbf{f} \\
\mathbf{0}
\end{array}\right\} .
$$

The internal DOFs $\mathbf{q}_{I}$ are dynamically condensed out using the bottom part of Eq. (6) by writing:

$$
-\mathbf{D}_{I, I}^{-1}\left(\mathbf{D}_{I, B L} \mathbf{q}_{B L}+\mathbf{D}_{I, B R} \mathbf{q}_{B R}+\mathbf{D}_{I, T L} \mathbf{q}_{T L}+\mathbf{D}_{I, T R} \mathbf{q}_{T R}+\mathbf{D}_{I, L} \mathbf{q}_{L}+\mathbf{D}_{I, R} \mathbf{q}_{R}+\mathbf{D}_{I, B} \mathbf{q}_{B}+\mathbf{D}_{I, T} \mathbf{q}_{T}\right)=\mathbf{q}_{I}
$$

and eliminating $\mathbf{q}_{I}$ from the remaining equations of Eq. (6), yielding the condensed equations of motion for the UC:

$$
\mathbf{D q}=\mathbf{f}
$$


with $\mathbf{D}$ the condensed dynamic stiffness matrix calculated per frequency $\omega$ for $i, j \in\{B L, B R, T L, T R, L, R, B, T\}$ as:

$$
\mathbf{D}_{i, j}=\hat{\mathbf{D}}_{i, j}-\hat{\mathbf{D}}_{i, I} \hat{\mathbf{D}}_{I, I}^{-1} \hat{\mathbf{D}}_{I, j}
$$

Combining the UC equations of motion (8) with the Bloch-Floquet boundary conditions (4) and (5) results in following EVP, depending on $\omega, \lambda_{x}$ and $\lambda_{y}$ [41]:

$$
\boldsymbol{\Lambda}_{L} \mathbf{D} \boldsymbol{\Lambda}_{R}\left\{\begin{array}{c}
\mathbf{q}_{B L} \\
\mathbf{q}_{L} \\
\mathbf{q}_{B}
\end{array}\right\}=\tilde{\mathbf{D}}\left(\omega, \lambda_{x}, \lambda_{y}\right)\left\{\begin{array}{c}
\mathbf{q}_{B L} \\
\mathbf{q}_{L} \\
\mathbf{q}_{B}
\end{array}\right\}=\mathbf{0}
$$

with $\tilde{\mathbf{D}}$ a general matrix depending on $\omega, \lambda_{x}$ and $\lambda_{y}$. This EVP is solved for the complex dispersion curves using the $\mu(\omega)$ approach, allowing frequency dependent properties to be used in the UC model. Considering time-harmonic motion, thus imposing real frequencies $\omega$, the above EVP takes the following form:

$$
\left[\tilde{\mathbf{D}}_{1}+\tilde{\mathbf{D}}_{2} \lambda_{x}+\tilde{\mathbf{D}}_{3} \lambda_{y}+\tilde{\mathbf{D}}_{4} \lambda_{x} \lambda_{y}+\tilde{\mathbf{D}}_{5} \lambda_{x}^{2}+\tilde{\mathbf{D}}_{6} \lambda_{y}^{2}+\tilde{\mathbf{D}}_{7} \lambda_{x}^{2} \lambda_{y}+\tilde{\mathbf{D}}_{8} \lambda_{x} \lambda_{y}^{2}+\tilde{\mathbf{D}}_{9} \lambda_{x}^{2} \lambda_{y}^{2}\right]\left\{\begin{array}{c}
\mathbf{q}_{B L} \\
\mathbf{q}_{L} \\
\mathbf{q}_{B}
\end{array}\right\}=\mathbf{0}
$$

with coefficient matrices $\tilde{\mathbf{D}}_{i}$. These coefficient matrices for general 2D UCs are reported for some specific combinations of $\lambda_{x}$ and $\lambda_{y}$ in a.o. [45, 46], considering UCs without damping. For completeness, in this work, the coefficient matrices for any combination of $\lambda_{x}$ and $\lambda_{y}$ are elaborated in Appendix A. EVP (11) can be reformulated as a polynomial EVP, as demonstrated by Mace et al. in [41], in case frequency and propagation direction $\theta$ are known and the ratio $\frac{\mu_{y}}{\mu_{x}}=\frac{L_{y}}{L_{x}} \tan (\theta)$ is rational. As proposed in [41], for a rational ratio $\frac{\mu_{y}}{\mu_{x}}=\frac{m_{2}}{m_{1}}$, the propagation constants can be expressed as $\mu_{x}=m_{1} \sigma$ and $\mu_{y}=m_{2} \sigma$. By introducing $\alpha=e^{\mathrm{i} \sigma}$ in Eq. (11), following polynomial EVP is obtained:

$$
\left[\tilde{\mathbf{D}}_{1}+\tilde{\mathbf{D}}_{2} \alpha^{m_{1}}+\tilde{\mathbf{D}}_{3} \alpha^{m_{2}}+\tilde{\mathbf{D}}_{4} \alpha^{m_{1}+m_{2}}+\tilde{\mathbf{D}}_{5} \alpha^{2 m_{1}}+\tilde{\mathbf{D}}_{6} \alpha^{2 m_{2}}+\tilde{\mathbf{D}}_{7} \alpha^{2 m_{1}+m_{2}}+\tilde{\mathbf{D}}_{8} \alpha^{m_{1}+2 m_{2}}+\tilde{\mathbf{D}}_{9} \alpha^{2 m_{1}+2 m_{2}}\right]\left\{\begin{array}{c}
\mathbf{q}_{B L} \\
\mathbf{q}_{L} \\
\mathbf{q}_{B}
\end{array}\right\}=\mathbf{0}
$$

By solving this EVP for various $\theta$ at given frequencies to $\sigma$, damped dispersion curves can be obtained. These represent the propagation constants $\mu$ or wavenumbers $k$ along the direction of propagation, calculated as:

$$
\mu=\sigma \sqrt{m_{1}^{2}+m_{2}^{2}} \quad \text { or } \quad k=\sigma \sqrt{\left(m_{1} / L_{x}\right)^{2}+\left(m_{2} / L_{y}\right)^{2}},
$$

against the frequency, indicating the wave modes in the structure. The solution of this polynomial EVP is obtained using Matlab's polynomial eigenvalue solver polyeig. In what follows, the presented UC method is applied to an LRM design including damping and used to asses the impact of damping in the different constituents. The numerically obtained dispersion curves are validated experimentally in Section 4. 


\section{Numerical unit cell analysis}

In this section an LRM design is introduced and the complex dispersion curves are analysed. The undamped dispersion curves along the IBC are solved by applying the classically used $\omega(\mu)$ approach. Next, the introduced $\mu(\omega)$ approach is applied to the undamped UC and compared to the $\omega(\mu)$ approach for three propagation directions. This is followed by an analysis of the influence of damping in resonator and host structure on the dispersion curves using the introduced $\mu(\omega)$ UC approach. Finally, the obtained results for the UC of the investigated LRM design are summarised for comparison with the experimental measurements.

\subsection{Locally resonant metamaterial design}

In order to apply the unit cell method introduced in previous section, in this work, an LRM is designed by adding resonant structures to a flat plate host structure. This section describes the metamaterial design, the materials and the geometry used. Establishing stop band behaviour requires the resonant additions to be spaced on a scale smaller than the wavelength to be affected, while the resonant motion should exert a non-zero net force on the host structure $[13,47]$. Stop band behaviour is desired for the NVH relevant out-of-plane bending motion of the structure [48]. As the metamaterial will be realised for experimental validation purposes, a realisable resonator design is envisaged that can be easily produced and straightforwardly modelled. Therefore, it has been decided to produce single-piece, singlematerial resonators by laser cutting from homogeneous plate material [20]. Since this procedure leads to extruded two dimensional shapes, in order to obtain resonant motion with a non-zero net force on the structure, the resonator is shaped as a cantilevered beam with an end-point mass and a support for the connection to the flat plate host structure using adhesive bonding, as depicted in Fig. 2a. The first bending mode of this resonator leads to a non-zero net force on the host structure in the out-of-plane direction (Fig. 2b). The geometry of the beam and end-point mass, together with the resonator material properties, allows the resonance frequency and the relative added mass to be tuned. Based on the resonator tuning and host structure properties, the subwavelength requirement is met by using proper spacing of the resonators.

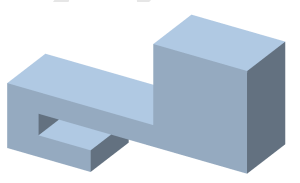

(a)

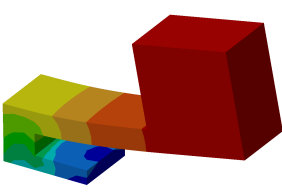

(b)

Figure 2 Resonant structure design. (a) Undeformed resonator and (b) first bending mode for clamped base.

As the unit cell method from Sec. 2 applies to two-dimensional planar structures, a flat host structure is selected. To keep the dynamic behaviour of the host structure straightforward, a $1 \mathrm{~mm}$ thick aluminium plate is used. The resonant structures are laser cut from a polymethyl methacrylate (PMMA) plate. The material properties of the aluminium 
and PMMA are retrieved by measuring and weighing a plate to obtain the density $\rho$ and applying a model updating procedure [49] with an FE model to obtain the Young's modulus $E$ and Poisson's ratio $v$. These material parameters are summarised in Tab. 1.

\begin{tabular}{lccc}
\hline Material & Young's modulus $\boldsymbol{E}$ & Poisson's ratio $\boldsymbol{\nu}$ & Density $\boldsymbol{\rho}$ \\
\hline \hline Aluminium & $69 \mathrm{GPa}$ & 0.33 & $2697 \mathrm{~kg} / \mathrm{m}^{3}$ \\
\hline PMMA & $4.85 \mathrm{GPa}$ & 0.31 & $1188 \mathrm{~kg} / \mathrm{m}^{3}$ \\
\hline
\end{tabular}

Table 1 Retrieved material parameters for host structure (aluminium) and resonator (PMMA).

With the obtained PMMA properties, the resonator geometry is tuned in order to obtain a resonance frequency of the first bending mode in the frequency range of interest up to $1000 \mathrm{~Hz}$. Besides the resonator frequency, a relative mass addition of less than $50 \%$ is maintained and the resonators should be compact enough to satisfy the subwavelength requirement. The obtained resonator dimensions are shown in Fig. 3, leading to a numerically predicted natural frequency for the first bending mode at $616 \mathrm{~Hz}$ for an FE model of the clamped base resonator consisting of 936 CHEXA8 solid elements [50], as shown in Fig. 2b. In order to satisfy the subwavelength requirement for flexural wave motion in the host structure in the targeted frequency range, the $\mathrm{UC}$ dimensions are chosen as $3 \times 3 \mathrm{~cm}$, leading to a relative mass addition of $39.8 \%$ with respect to the bare host structure UC. The resonators are added to the aluminium plate by means of adhesive bonding, using a Loctite ${ }^{\circledR} 406^{\mathrm{TM}}$ contact adhesive, and oriented with the long side of the beam along the $x$-direction (Fig. 4a). Due to its non-symmetric shape, contrary to typically analysed spring-mass resonators, fixing the orientation of the resonator is important as it can, depending on the coupling between resonant motion and wave propagation for a certain direction, lead to directionally dependent attenuation performance. By fixing the resonator orientation, a proper comparison can be made between the numerical predictions and experimental measurements of the periodic metamaterial structure. The convention of the IBC in the reciprocal lattice, along which the undamped dispersion curves are generally calculated using the $\omega(\mu)$ approach, is shown for this UC in Fig. $4 \mathrm{~b}$ and is given by $\operatorname{Re}\left(\mu_{x}, \mu_{y}\right): \mathrm{O}-\mathrm{A}-\mathrm{B}-\mathrm{C}-\mathrm{O} \mapsto(0,0),(\pi, 0),(\pi, \pi),(0, \pi),(0,0)$.
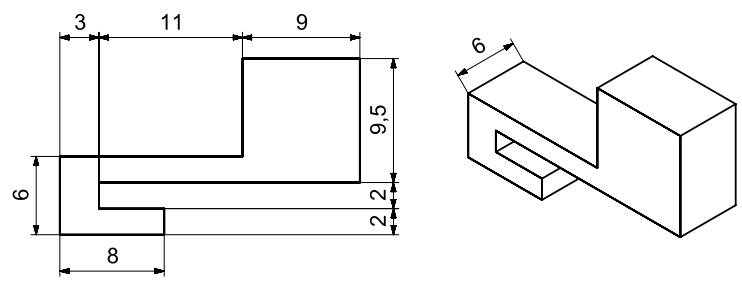

Figure 3 Geometry of the resonant structures, dimensions in $\mathrm{mm}$.

Since this work investigates the UC analysis approach including damping, damping is included in the UC model of the LRM. The UC approach introduced in Sec. 2 allows a variety of (frequency dependent) properties and damping 


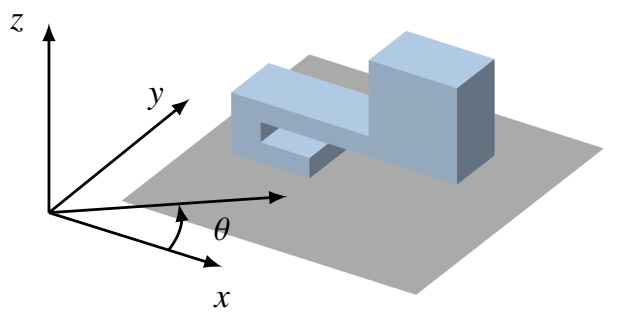

(a)

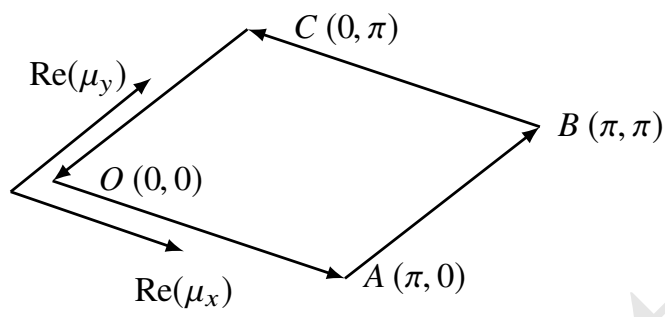

(b)

Figure 4 Unit cell of the designed locally resonant metamaterial. (a) Unit cell orientation, with propagation direction $\theta$ and (b) IBC in reciprocal lattice.

models to be included in the frequency dependent dynamic stiffness matrix. However, in this work, the main interest is to include the presence of damping in both metamaterial constituents, which is often neglected, rather than providing accurate damping models. For the introduced metamaterial design, only intrinsic damping is considered in the resonator and host structure. This is accounted for in the FE model of the UC from Eq. (1) by considering a complex stiffness matrix $\mathbf{K}$ in the dynamic stiffness matrix as follows [39, 51]:

$$
\mathbf{K}=\sum_{q=1}^{n_{q}}\left(\mathbf{K}_{q}+\mathrm{i} \mathbf{K}_{q} \eta_{q}\right),
$$

with $\mathbf{K}_{q}$ the real stiffness matrix contribution of the $q$-th finite element, $\eta_{q}$ the corresponding structural damping coefficient, $\sum$ the stiffness matrix assembly operator and $n_{q}$ the number of elements in the UC. In general $\mathbf{K}$ can be frequency dependent, yet here a constant damping coefficient is used per material. A structural damping coefficient is estimated for the PMMA of the resonant structures. Using an SLDV measurement of the structural response of five different resonators, glued at the base to a shaker, application of the half power bandwidth method at the resonance frequency results in an average structural damping coefficient of $\eta_{\text {res }}=5 \%$ [52], with an average resonance frequency of $624 \mathrm{~Hz}$. In this way, a possibly small damping contribution of the same thin adhesive layer is accounted for. Similarly, for the aluminium, the same method is applied to the resonance peaks around the tuned resonator frequency for a roving hammer measurement of the host structure plate, yielding a structural damping coefficient of $\eta_{\text {host }}=0.28 \%$. The eventual FE model of the $3 \times 3 \mathrm{~cm} \mathrm{UC} \mathrm{(Fig.} 4 \mathrm{a}$ ) for the dispersion curve calculations consists of 936 CHEXA8 solid elements for the PMMA resonator and 930 CQUAD4 shell elements for the aluminium plate. The glue layer between resonator and host structure is not modelled explicitly, but mesh connectivity is obtained by direct elimination of the interfacing DOFs and additional damping contributions are accounted for in the measured damping coefficient, since the same adhesive is used for the manufactured sample. 


\subsection{Undamped unit cell}

\subsection{1. $\omega(\mu)$ approach}

To determine the stop band frequency range of the undamped LRM, the undamped dispersion curves are calculated along the IBC using the $\omega(\mu)$ approach and are presented in Fig. 5, showing the calculated frequencies against the real propagation vector along the IBC. In this diagram, dispersion curves for different wave types emerge; shear, longitudinal and flexural waves are observed. The flexural wave type shows up as the most dispersive type and is the targeted wave type for stop band behaviour. A resonance based stop band is observed for the flexural waves around the tuned frequency of the resonant additions, between 597 and $656 \mathrm{~Hz}$, since no freely propagating flexural wave solutions are found in that frequency range along the entire IBC.

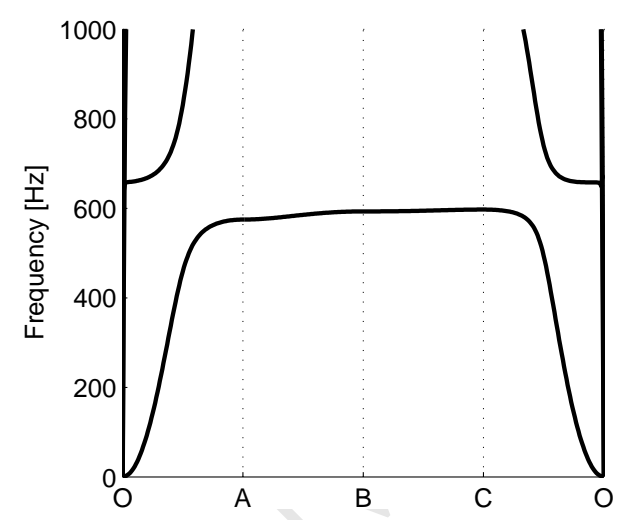

Figure 5 Dispersion curves along the IBC obtained with the $\omega(\mu)$ approach for the undamped unit cell.

In order to analyse the wave solutions inside the IBZ, the $\omega(\mu)$ approach is applied to the undamped UC for propagation vectors along various propagation directions $\theta=0^{\circ}, 45^{\circ}$ and $90^{\circ}$. The obtained dispersion curves for these three directions are shown in Fig. 6.

In these dispersion curves, directional band gaps are found for the flexural wave mode between 575 and $656 \mathrm{~Hz}$, 593 and $656 \mathrm{~Hz}$ and 597 and $656 \mathrm{~Hz}$ for the directions $\theta=0^{\circ}, 45^{\circ}$ and $90^{\circ}$ respectively. The difference in band gap widths for different propagation directions can be attributed to different distances between resonators on the one hand and different interaction between the flexural waves and the resonators on the other hand. The latter can indicate a different efficiency of the resonant mass for different resonator orientations, with the $\theta=0^{\circ}$ direction being more favourable. Differences also show up in the interaction between in-plane and out-of-plane modes after the band gap. It is known that the motion of the structure before the stop band consists of in-phase motion between resonator and host structure, while after the stop band these modes are repeated at higher frequencies with out-of-phase motion [13]. In Fig. 6, the interaction between the repeated flexural wave branch with the in-plane longitudinal and shear wave modes is observed to vary with the propagation direction for the structure at hand. For the $\theta=0^{\circ}$ direction, veering takes place between the longitudinal and bending mode, for the $\theta=90^{\circ}$ direction between shear and bending, while 


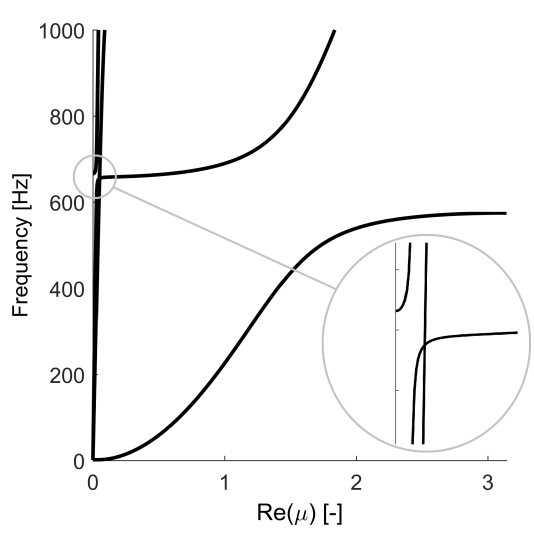

(a)

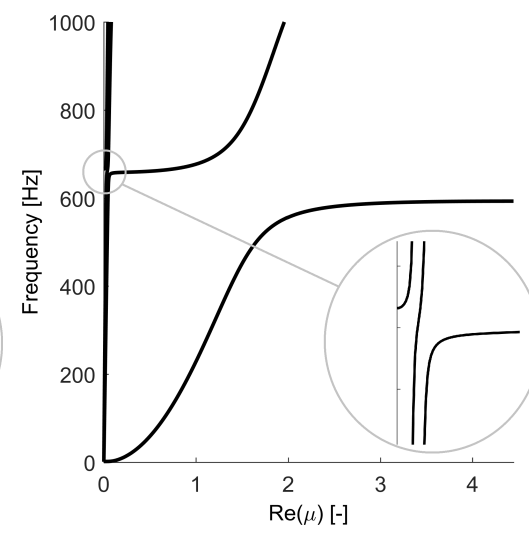

(b)

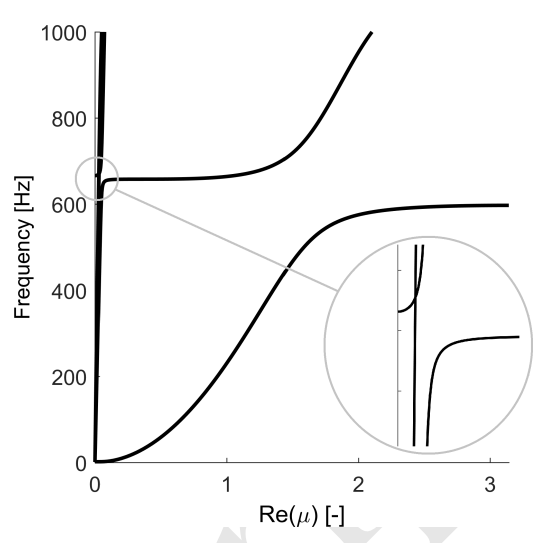

(c)

Figure 6 Dispersion curves for propagation directions $\theta=0^{\circ}$ (a), $45^{\circ}$ (b) and $90^{\circ}$ (c) calculated with the $\omega(\mu)$ approach for the undamped unit cell.

for the $\theta=45^{\circ}$ direction, both longitudinal and shear modes veer with the bending mode. This can be explained by a stronger interaction between the out-of-plane flexural waves and the first bending mode of the considered resonator, for an excitation of the resonator basis with a component in the $x$-direction of the resonator.

\subsection{2. $\omega(\mu)$ and $\mu(\omega)$ approach}

The dispersion curves are now calculated using the $\mu(\omega)$ approach from Sec. 2 along the three propagation directions $\theta=0^{\circ}, 45^{\circ}$ and $90^{\circ}$ for the undamped $\mathrm{UC}$ in the frequency range from 1 to $1000 \mathrm{~Hz}$ with steps of $1 \mathrm{~Hz}$ and compared with the results from the $\omega(\mu)$ approach. The solutions for the propagation constants $\mu$ are found inside the first Brillouin zone, implying $\operatorname{Re}(\mu) \in[-\pi, \pi]$ for $\theta=0^{\circ}, 90^{\circ}$ and $\operatorname{Re}(\mu)[-\sqrt{2} \pi, \sqrt{2} \pi]$ for $\theta=45^{\circ}$. The results are shown in Fig. 7 together with the corresponding results from the $\omega(\mu)$ approach.

An excellent agreement is found between the $\omega(\mu)$ and $\mu(\omega)$ approach for the undamped UC for all propagation directions. The same interaction is obtained between the in- and out-of-plane modes in the structure at hand. Inspecting the band gap frequency ranges, where no solutions are obtained using the $\omega(\mu)$ approach, solutions with a non-zero real part of the propagation vector are now found using the $\mu(\omega)$ approach.

To gain further insight into the results from the $\mu(\omega)$ approach, the dispersion curves for the three propagation directions are shown in the complex $(\mu, f)$-space in Fig. 8. These results are repeated in Fig. 9, viewed from above, representing the complex $\mu$-plane for the considered frequency range. For completeness both left and right going wave solutions are shown, corresponding to $\operatorname{Re}(\mu)>0$ and $\operatorname{Re}(\mu)<0$ respectively. This corresponds to taking slices throughout the first Brillouin zone for the considered propagation directions. The real, propagating part of the obtained propagation constant is limited to the bounds of the first Brillouin zone, as the propagating part of the solution is $2 \pi$-periodic in the wave domain. The imaginary, decaying part of the propagation constant is not subjected to this 


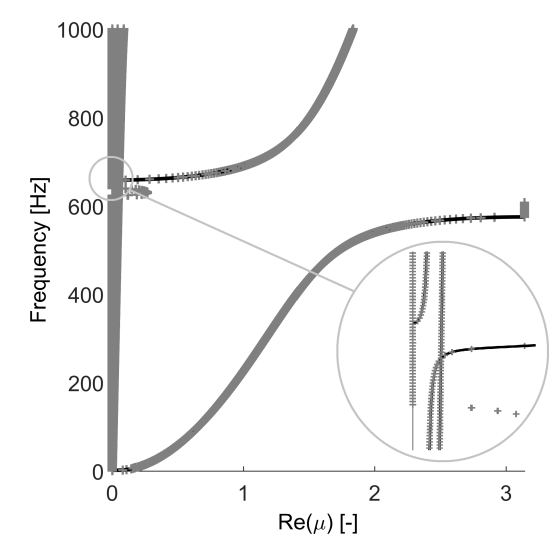

(a)

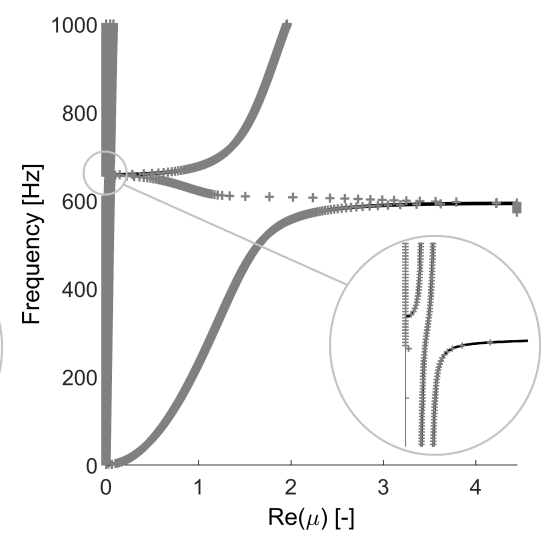

(b)

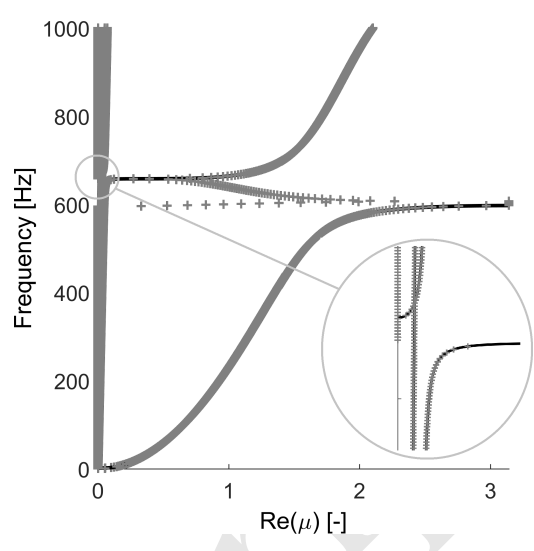

(c)

Figure 7 Comparison of dispersion curves for propagation directions $\theta=0^{\circ}$ (a), $45^{\circ}$ (b) and $90^{\circ}$ (c) calculated with the $\omega(\mu)(-)$ and $\mu(\omega)(+)$ approach for the undamped unit cell.

periodicity. For sake of clarity these solutions are limited to those lying closest to the origin, $\operatorname{Im}(\mu) \in[-\pi, \pi]$, in which the relevant results for the considered metamaterial structure are found. Solutions with a larger imaginary part are also found, part of which are related to the polynomial EVP and FE discretisation [41].

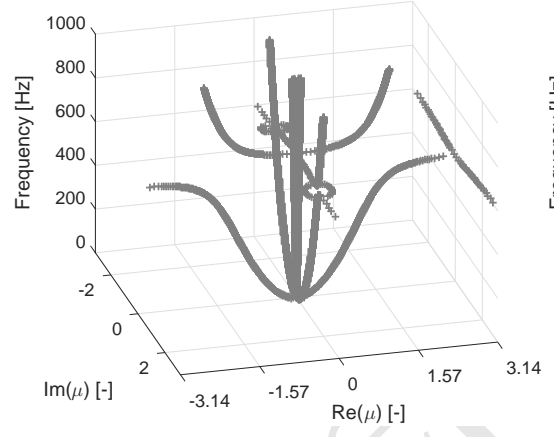

(a)

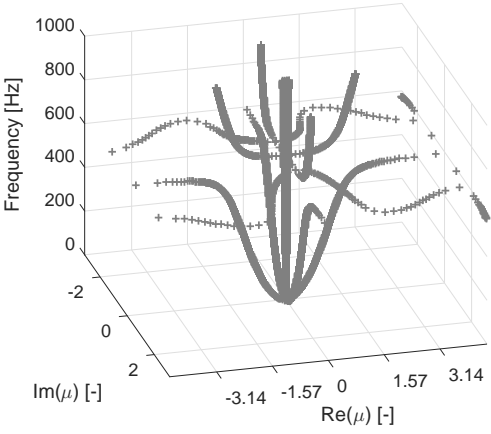

(b)

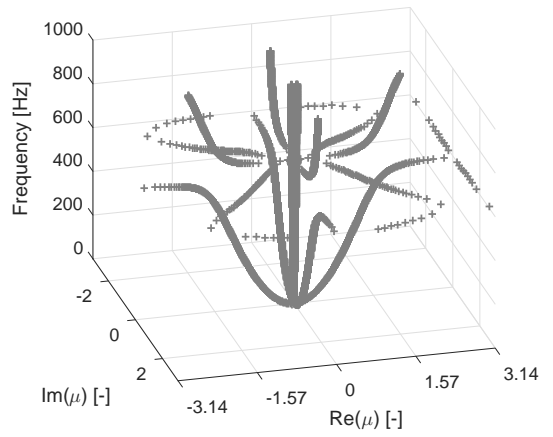

(c)

Figure 8 Complex dispersion curves for propagation directions $\theta=0^{\circ}$ (a), $45^{\circ}$ (b) and $90^{\circ}$ (c) calculated using the $\mu(\omega)$ approach for the undamped unit cell.

As can be observed in Fig. 8, no freely propagating flexural wave solutions, thus pure real propagation vectors, are found inside the band gaps predicted before with the $\omega(\mu)$ approach. Outside the band gap frequency ranges, the flexural wave solutions occur in pairs of freely propagating, real propagation constants and evanescent, imaginary propagation constants. As no free wave propagation is allowed in the band gap frequency zones, imposing real frequencies causes the propagation constants to occur in either imaginary or complex $\mu$ solution pairs. Similar to the observations by Laude et al. [53] for the complex band structure of phononic crystals, the maximum of the propagating 


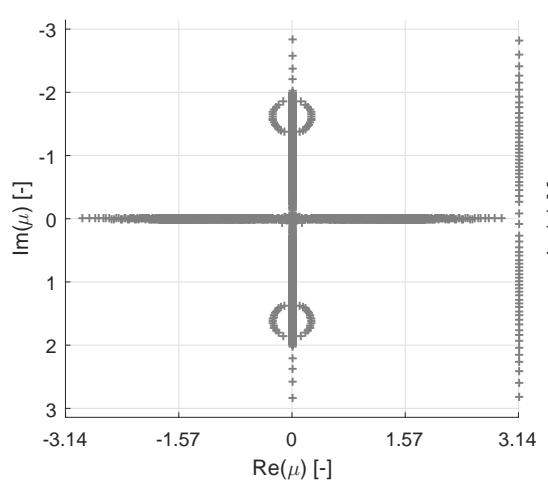

(a)

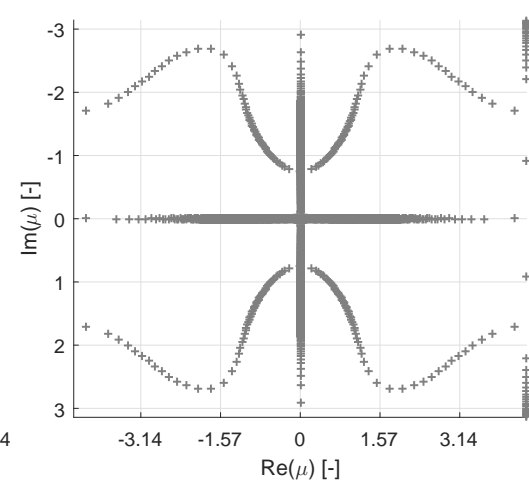

(b)

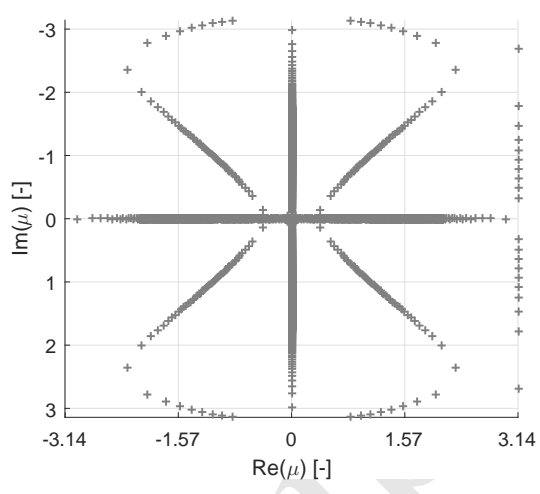

(c)

Figure 9 Top view of the complex dispersion curves for propagation directions $\theta=0^{\circ}$ (a), $45^{\circ}$ (b) and $90^{\circ}$ (c) calculated using the $\mu(\omega)$ approach for the undamped unit cell.

solution branch at the bottom of the stop band, at $\operatorname{Re}(\mu)=\pi$, transitions into a complex $\mu$ solution to maintain the amount of wave solutions inside the stop band. For Bragg interference stop bands, this complex solution transitions towards the minimum of the repeated propagating branch starting after the stop band, at $\operatorname{Re}(\mu)=\pi$. Similarly, for the here considered resonance based stop band, the maximum and minimum of the propagating branches respectively before and after the stop band, are connected through a sequence of bifurcating complex $\mu$ solutions. It is noted that, due to the adopted convention $e^{\mathrm{i} \mu}$ in Sec. 2, the complex propagation constants with real and imaginary parts of opposite sign represent spatially decaying solutions. The complex solution pairs in the opposing quadrants correspond to flexural waves of exponentially increasing amplitude. For this case, the complex transition of the maximum of the propagating branch below the stop band at $\operatorname{Re}(\mu)=\pi$ manifests as a bifurcation of the propagating solutions originating at $\operatorname{Re}(\mu)=0$ and $\operatorname{Re}(\mu)=2 \pi$ into two complex conjugate solutions, of which the exponentially increasing solution corresponds to the decaying solution originating from $\operatorname{Re}(\mu)=2 \pi$. As different interactions are found between in- and out-of-plane modes for different directions, the shape of the observed complex solution pairs also varies per direction. These complex $\mu$ solutions are further discussed in the upcoming sections, when damping is introduced.

In conclusion, an excellent agreement is obtained between the complex $\mu$ solutions from the presented $\mu(\omega)$ approach and the real $\omega$ solutions from the $\omega(\mu)$ approach for the undamped metamaterial UC, while the representation of the complex $\mu$ solutions allows to understand the complex dispersion curves in and around the stop band.

\subsection{Damped unit cell}

When damping is introduced, the free wave propagation considered in the $\omega(\mu)$ approach no longer suffices to capture the now complex dispersion curves. Structural damping values of $\eta_{\text {res }}=5 \%$ and $\eta_{\text {host }}=0.28 \%$ have been obtained for the PMMA and aluminium respectively, indicating that rather highly damped resonators are added to the quasi undamped host structure. Consequently, the presented $\mu(\omega)$ unit cell approach is used to analyse the complex 
$\mu$ dispersion curves for the considered metamaterial structure including damping. To verify the damping contribution of the different constituting elements, first damping is considered in the resonator and the host structure separately, followed by their combined presence.

\subsection{1. $\mu(\omega)$ approach, influence of damping in the resonator}

In this first section, three different structural damping coefficients $\eta_{r e s}=1 \%, 2 \%$ and $5 \%$ are considered for the resonator, with the highest value corresponding to the experimentally retrieved result, while the host structure remains undamped. The $\mu(\omega)$ approach is used to calculate the complex $\mu$ dispersion curves for the three propagation directions $\theta=0^{\circ}, 45^{\circ}$ and $90^{\circ}$. The complex $\mu$ dispersion curves are shown in Fig. 10 and repeated in Fig. 11, viewed from above, with the solutions for $\eta_{\text {res }}=1 \%$ represented by light gray pluses, $\eta_{\text {res }}=2 \%$ by dark gray diamonds and $\eta_{\text {res }}=5 \%$ by black circles.

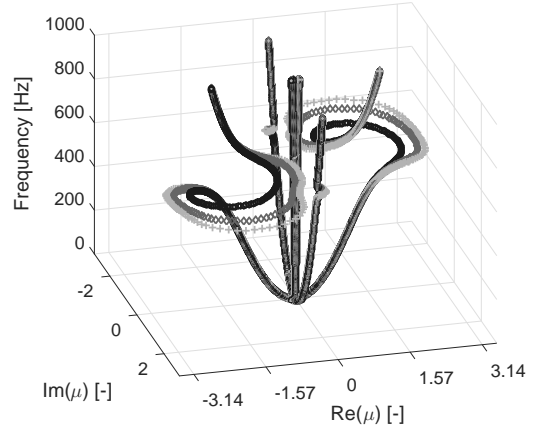

(a)

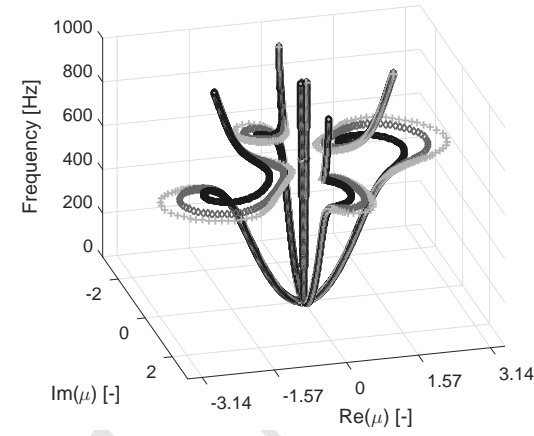

(b)

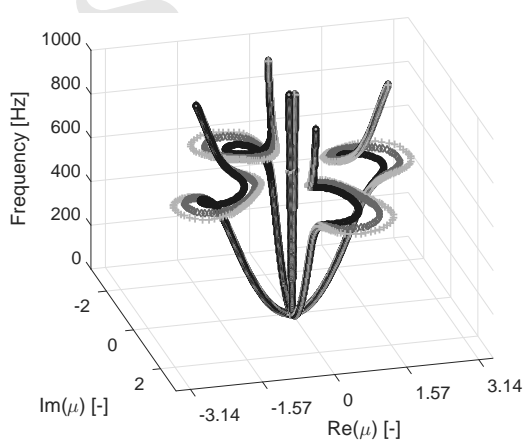

(c)

Figure 10 Influence of damping in the resonator for propagation directions $\theta=0^{\circ}$ (a), $45^{\circ}$ (b) and $90^{\circ}$ (c), for $\eta_{\text {res }}=1 \%(+), 2 \%$ $(\diamond)$ and $5 \%(\circ)$, with undamped host structure, calculated using the $\mu(\omega)$ approach.

While for the undamped results the complex $\mu$ solutions only occur inside the band gap zones, where no freely propagating solutions are allowed, the unit cell with damped resonator leads to all-complex $\mu$ dispersion curves for the given real frequencies. Consequently, the notion of a stop band as a frequency region without free wave propagation fades. Compared to the obtained results with the results for the undamped unit cell, no notable difference is observed far away from the stop band region. However, in the vicinity of and inside the band gap, the complex bifurcating solutions inside the band gap for the undamped unit cell are now replaced by a complex $\mu$ loop, causing a gradual transition between the previously freely propagating flexural wave solutions before and after the stop band. The same effect occurs for the previously purely evanescent solutions, yet in this case only the complex propagation constants with $\operatorname{Re}(\mu)>0$ and $\operatorname{Im}(\mu)<0$ or $\operatorname{Re}(\mu)>0$ and $\operatorname{Im}(\mu)<0$, represent spatially decaying flexural waves. The stop band now becomes a zone of strongly attenuated wave propagation.

Upon closer inspection of the complex $\mu$ flexural solution transitions, it can be seen that their covered frequency range exceeds the undamped band gap frequency range. The addition of damping thus also broadens the frequency 


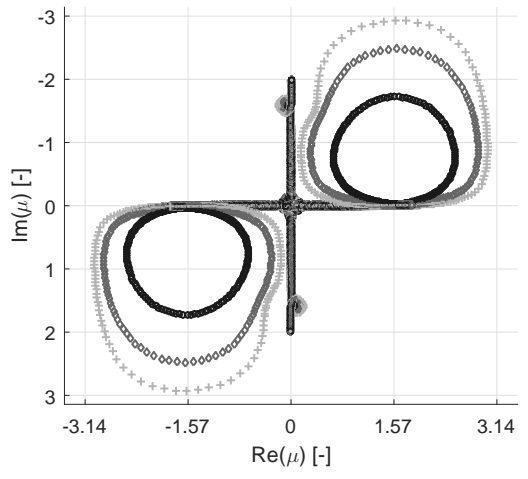

(a)

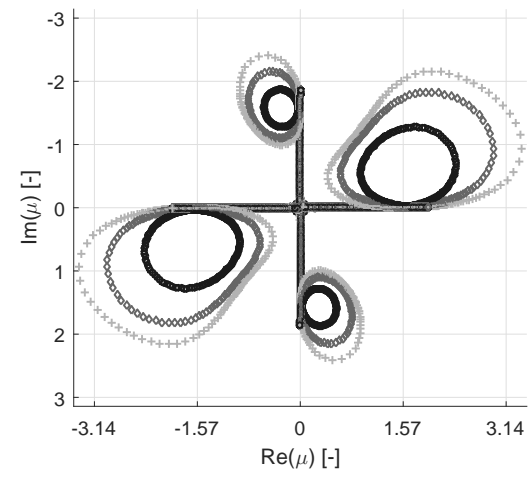

(b)

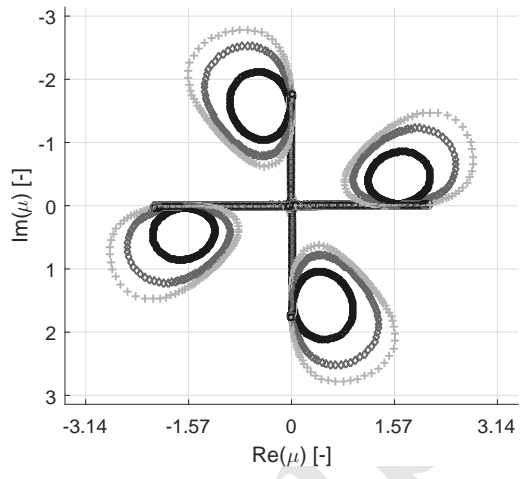

(c)

Figure 11 Influence of damping in the resonator for propagation directions $\theta=0^{\circ}$ (a), $45^{\circ}$ (b) and $90^{\circ}$ (c), for $\eta_{\text {res }}=1 \%$ ( + ), $2 \%$ $(\diamond)$ and $5 \%$ (०), with undamped host structure, calculated using the $\mu(\omega)$ approach.

range of attenuation for the targeted wave type. Another effect of the complex transition loop is found in the coverage of the real, propagating part of the wave solution of the first Brillouin zone around the band gap. While for the undamped case, the flattened flexural branch before and after the stop band zone causes ample presence of modes with respectively in-phase and out-of-phase motion between resonator and host structure, the complex $\mu$ transition loop causes part of these modes to vanish and the edge of the Brillouin zone is no longer attained in that frequency region. Consequently in the case of damping, the IBC no longer captures the phenomena around the stop band frequency range. This is in accordance with the remarks in $[32,37]$ regarding the insufficiency of the IBC in presence of damping.

By increasing the damping in the resonator, the radius of the complex $\mu$ loops decreases, while their covered frequency range increases. Since the decreasing radius causes the imaginary part of the propagation constant to reduce inside the stop band frequency range, the spatial attenuation and hence the peak attenuation performance of the metamaterial decreases. On the other hand, the reduction of the transition loop size with increasing damping causes more of the previously present freely propagating flexural modes before and after the stop band to disappear, while the increasing frequency range of the complex $\mu$ loops broadens the frequency range of attenuation. By increasing the damping in the resonator, while a clear decrease in attenuation performance occurs in the original stop band frequency range, the frequency range of attenuation is widened. These findings agree well with previous observations for one-dimensional and finite two-dimensional structures in [13] and are illustrated for the $\theta=45^{\circ}$ propagation direction in Fig. 12, by showing the propagating part $\operatorname{Re}(\mu)$ of the dispersion curves for flexural, longitudinal and shear modes, coloured according to the magnitude of the decaying part of the propagation constant $|\operatorname{Im}(\mu)|$, together with the stop band frequency ranges from Sec. 3.2.1 for the three damping coefficients $\eta_{r e s}=1 \%, 2 \%$ and $5 \%$. It is noted that, where the band gap width varies for different propagation directions, the same damping value tends to have a stronger effect on the smaller band gaps yielding a smaller complex $\mu$ loop and thus a lower attenuation performance. 


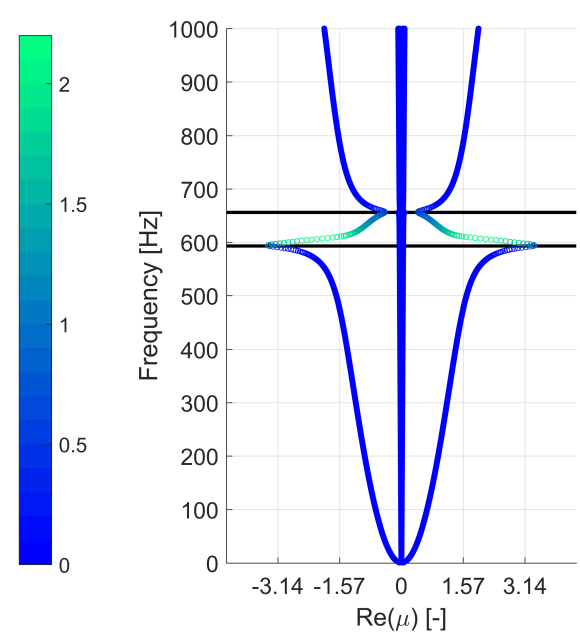

(a)

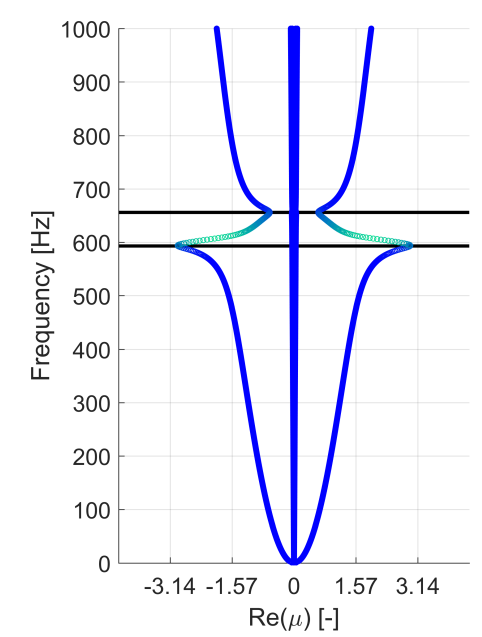

(b)

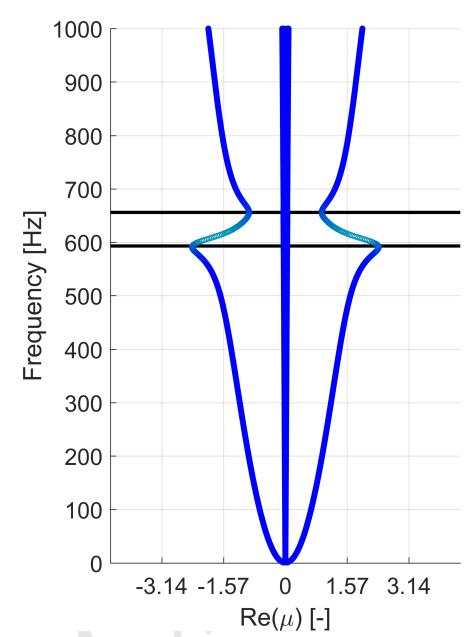

(c)

Figure 12 Dispersion curves for $\eta_{r e s}=1 \%$ (a), $2 \%$ (b) and $5 \%$ (c) obtained with the $\mu(\omega)$ approach for propagation direction $\theta=45^{\circ}$, coloured according to $|\operatorname{Im}(\mu)|$, with solid horizontal lines indicating the band gaps predicted using the $\omega(\mu)$ approach.

\subsection{2. $\mu(\omega)$ approach, influence of damping in the host structure}

Although the structural damping coefficient $\eta_{h o s t}=0.28 \%$ of the aluminium plate host structure is considerably lower than the $\eta_{\text {res }}=5 \%$ for the resonant structures, the effect of damping in the host structure on the stop band behaviour is analysed separately in this section. To this end three different damping coefficients $\eta_{\text {host }}=0.28 \%, 1 \%$ and $5 \%$ are considered for the host structure, with the lowest value corresponding to the experimentally obtained result, while the resonator remains undamped. The complex dispersion curves, obtained through the $\mu(\omega)$ unit cell method, are shown in Fig. 13 and 14. In these figures, $\eta_{\text {host }}=0.28 \%$ is represented by light gray pluses, $\eta_{\text {host }}=1 \%$ by dark gray diamonds and $\eta_{\text {host }}=5 \%$ by black circles.

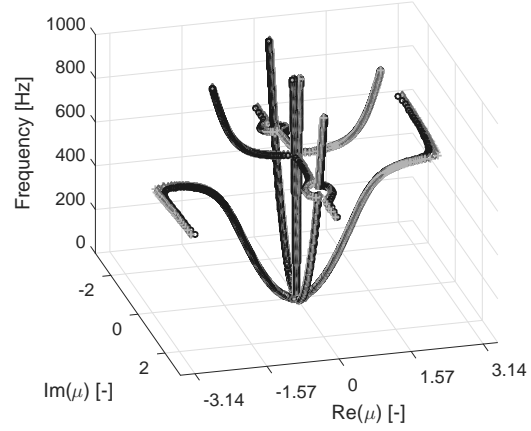

(a)

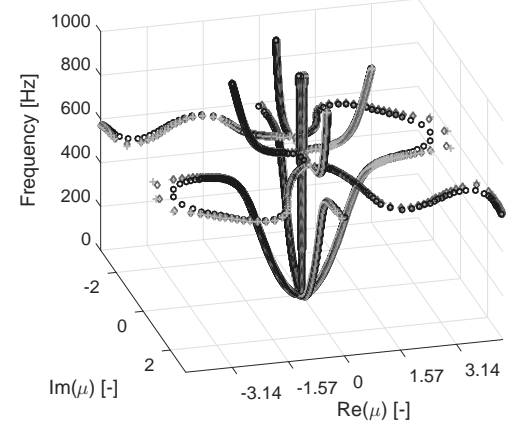

(b)

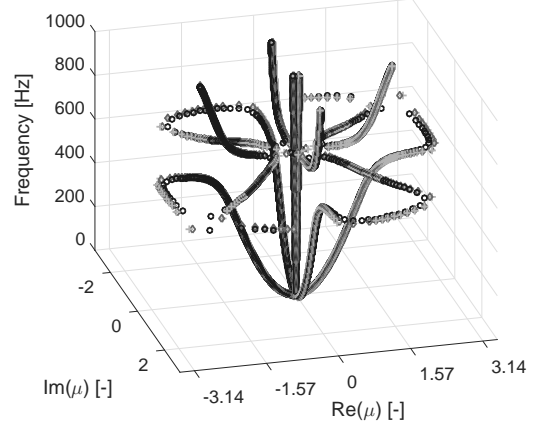

(c)

Figure 13 Influence of damping in the host structure for propagation directions $\theta=0^{\circ}$ (a), $45^{\circ}$ (b) and $90^{\circ}$ (c), for $\eta_{\text {host }}=0.28 \%$ $(+), 1 \%(\diamond)$ and $5 \%(\circ)$, with undamped resonator, calculated using the $\mu(\omega)$ approach. 


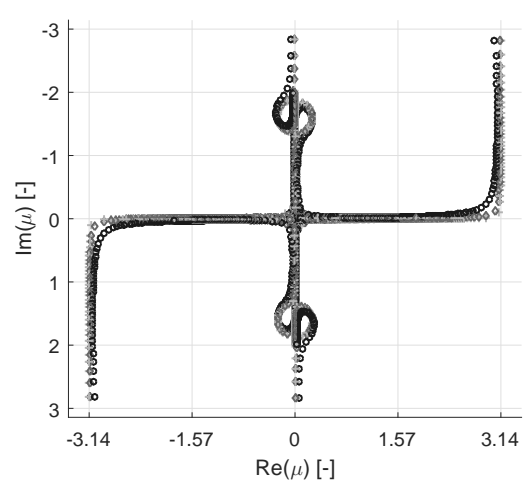

(a)

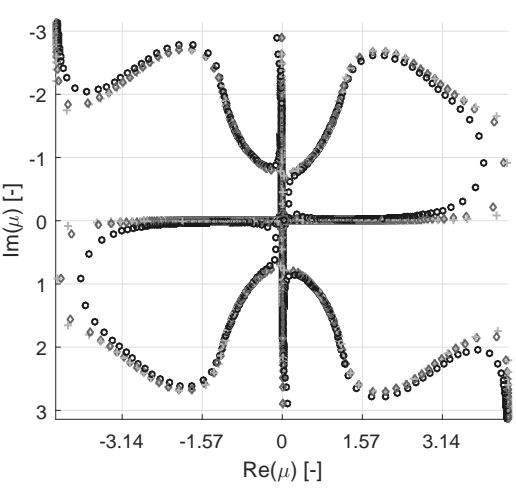

(b)

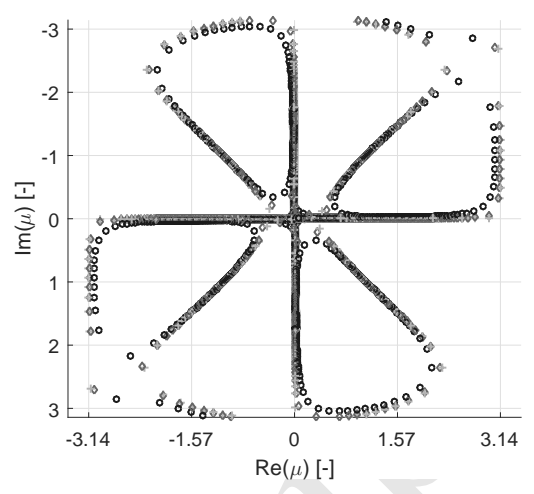

(c)

Figure 14 Influence of damping in the host structure for propagation directions $\theta=0^{\circ}$ (a), $45^{\circ}$ (b) and $90^{\circ}$ (c), for $\eta_{\text {host }}=0.28 \%$ $(+), 1 \%(\diamond)$ and $5 \%(\circ)$, with undamped resonator, calculated using the $\mu(\omega)$ approach.

The presence of damping again renders all propagation constants complex, yet the differences with the undamped unit cell are limited in the considered frequency range. As before, the addition of damping causes the previously freely propagating and evanescent wave branches to turn into the complex plane, causing a gradual transition between the region before and after the band gap zone. Compared to the undamped results from Fig. 8 and 9, the addition of damping more clearly reveals the transition into complex solutions at the maximum of the propagating branches before the stop band in both propagation directions, which is now gradually smoothed. The effect is however considerably less outspoken than the contribution of damping in the resonators, for the considered metamaterial structure. For the same damping coefficient magnitude, damping in only the resonators leads to clear complex $\mu$ solution loops, while for damping in only the host structure merely the onset of this gradual complex transition is found.

Apart from the damping influence in the vicinity of the stop band frequency range, another effect is now also observed outside of this region. With increasing damping, the dispersion curves undergo a counter-clockwise tilting in the complex $\mu$-plane. This can be explained by the used constant structural damping coefficient. In [54] it is shown that the damping coefficient $\eta$ for bending waves in a plate is related to the complex wavenumber $k$ as:

$$
\eta=\left|\frac{\operatorname{Im}\left(k^{4}\right)}{\operatorname{Re}\left(k^{4}\right)}\right| .
$$

For low $\eta$, thus lightly damped structures, the decaying part of the wavenumber $\operatorname{Im}(k) \ll \operatorname{Re}(k)$ and above relation simplifies to:

$$
\eta \approx 4\left|\frac{\operatorname{Im}(k)}{\operatorname{Re}(k)}\right|
$$

As a constant damping coefficient $\eta$ is used, above fixed relation between real and imaginary parts of the wavenumber affects the dispersion curves accordingly by tilting in the complex $\mu$-plane. A similar effect is observed for the other wave modes. This damping effect on the wave propagation in the host structure is superimposed with the complex transition around the stop band frequency range. 


\subsection{3. $\mu(\omega)$ approach, influence of damping in the host structure and resonator}

In the previous sections, the effects on the complex dispersion curves of damping in resonator and host structure have been discussed separately. Since damping is present in both constituting parts, damping is now considered in both host structure and resonator. The damping in the host structure is again varied between $\eta_{\text {host }}=0.28 \%, 1 \%$ and $5 \%$, while the experimentally determined structural damping value $\eta_{\text {res }}=5 \%$ is used for the resonant structure. The calculated complex $\mu$ dispersion curves for the three propagation directions are shown in Fig. 15 and 16.

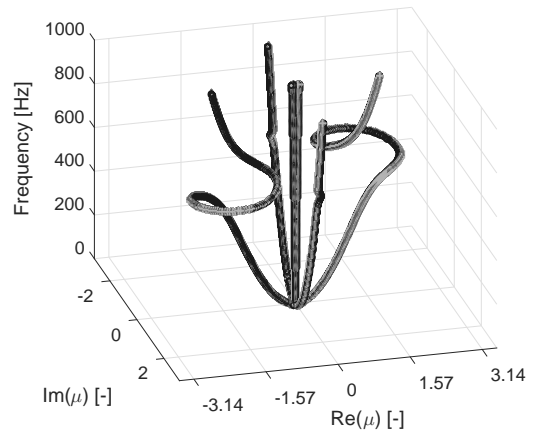

(a)

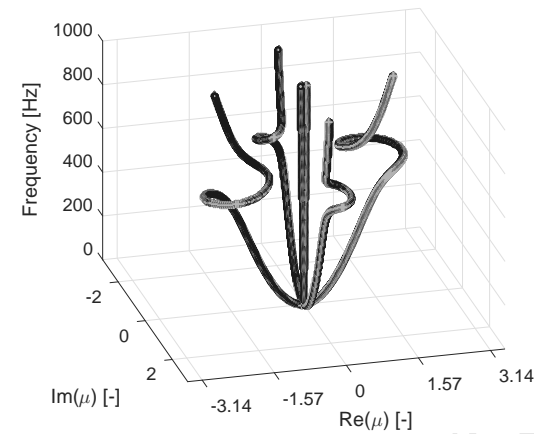

(b)

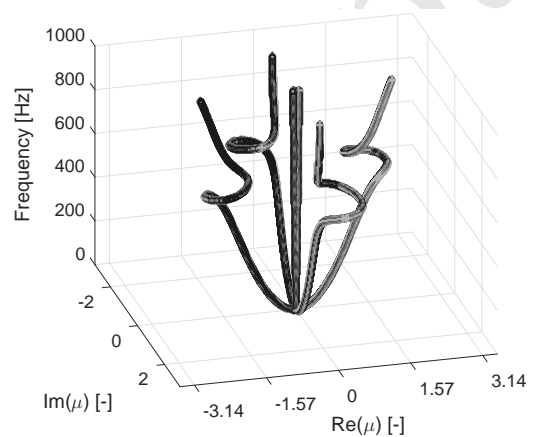

(c)

Figure 15 Influence of damping in the resonator for propagation directions $\theta=0^{\circ}$ (a), $45^{\circ}$ (b) and $90^{\circ}$ (c), for $\eta_{\text {host }}=0.28 \%$ ( + ), $1 \%(\diamond)$ and 5\% (०), with $\eta_{\text {res }}=5 \%$ for the resonator, calculated using the $\mu(\omega)$ approach.

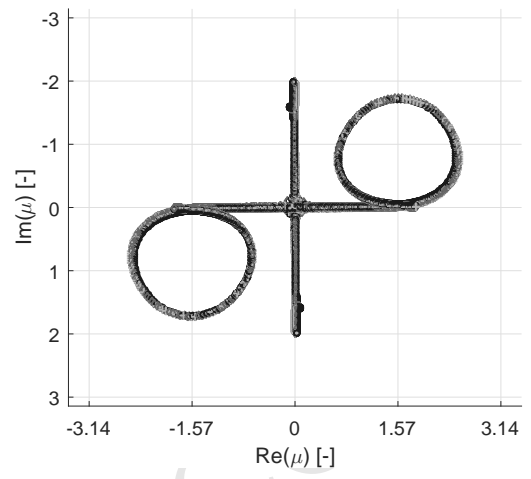

(a)

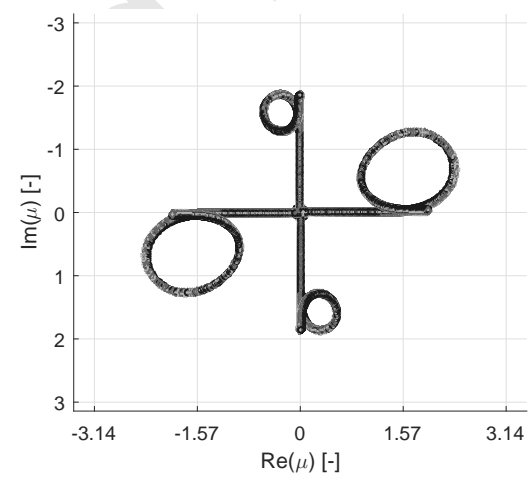

(b)

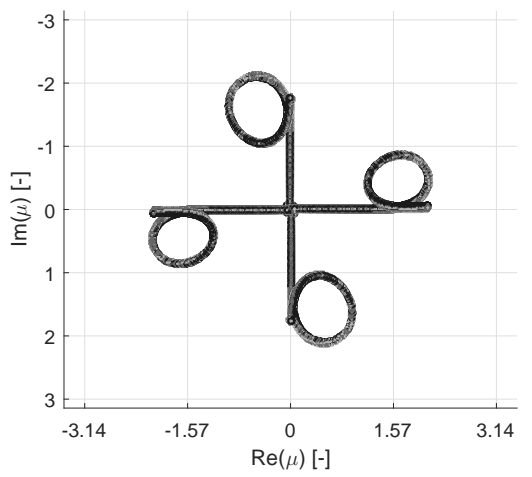

(c)

Figure 16 Influence of damping in the resonator for propagation directions $\theta=0^{\circ}$ (a), $45^{\circ}$ (b) and $90^{\circ}$ (c), for $\eta_{\text {host }}=0.28 \%$ ( + ), $1 \%(\diamond)$ and $5 \%(\circ)$, with $\eta_{\text {res }}=5 \%$ for the resonator, calculated using the $\mu(\omega)$ approach.

As expected from the results obtained with damping in the host structure only, the additional influence of damping in the host structure is limited for the considered structure as compared to the influence of damping in the resonator. A slight additional decrease of the complex solution loop radius is observed together with a slight broadening of the 
covered frequency range. Furthermore, the counter-clockwise tilting of the dispersion curves over the entire frequency range, originating from the damping in the host structure, is added and increases with damping.

\subsection{Summary and dispersion curves for experimental validation}

Based on the preceding analyses, the effects of the presence of damping in the different constituents on the attenuation performance of the considered plate-type locally resonant metamaterial, can be summarized as follows:

- Damping in the resonator causes the previously freely propagating flexural wave solutions before and after the stop band to close by means of a complex $\mu$ solution loop. $\operatorname{Im}(\mu)$ increases around the stop band with increasing damping, broadening the zone of attenuation, while $\operatorname{Im}(\mu)$ decreases inside the stop band, reducing peak attenuation performance. Further away from the stop band, the flexural wave attenuation is largely unaffected as the $\operatorname{Im}(\mu)$ increase is limited.

- Damping in the host structure leads to only the onset of the complex $\mu$ solution loop formation around the stop band, leaving the attenuation in the stop band largely unaffected. Outside the stop band, however, the attenuation of the flexural wave mode is more obviously increased with increasing damping as the dispersion curves rotate into the complex plane according to the loss factor of the host structure.

- Damping in both resonator and host structure combines the above, mainly complementary, effects.

For the considered LRM, as the damping coefficient of the aluminium plate host structure is considerably lower than for the PMMA resonators, the main damping contribution is coming from the resonant structures, affecting the region in and around the stop band zone. Consequently, for this type of metamaterial design, the damping in the resonant additions can be well chosen to obtain a certain desired attenuation performance over a broadened frequency range, rather independent of the damping in a lightly damped host structure.

The results of the $\mu(\omega)$ unit cell analysis for the eventually realised metamaterial structure including the measured damping coefficients $\eta_{\text {res }}=5 \%$ and $\eta_{\text {host }}=0.28 \%$ are presented in Fig. 17 for the three propagation directions $\theta=0^{\circ}, 45^{\circ}$ and $90^{\circ}$. In these dispersion curves only the flexural wave solutions are presented, this time by means of the real, propagating part of the wavenumber $\operatorname{Re}(k)=k_{\text {prop }}$, coloured according to the attenuation, expressed by magnitude of the imaginary, decaying part of the wavenumber $|\operatorname{Im}(k)|$, for which the experimental dispersion curves will be acquired in the following section.

For the calculated dispersion curves, the analysed frequency range is extended to $1250 \mathrm{~Hz}$, in order to take into account the influence of the second mode of the resonant structure with a natural frequency at $1214 \mathrm{~Hz}$ (Fig. 18b). Contrary to the often considered simple mass-spring type resonators, the realisable resonator has more than one eigenmode that can allow band gap behaviour to be introduced. While the first mode (Fig. 18a), for which the resonator design is intended, is an outspoken out-of-plane bending mode, the second mode is an in-plane bending mode. In this mode, the resonator mass exerts a sideways swaying motion in the $y$-direction, involving also a smaller 


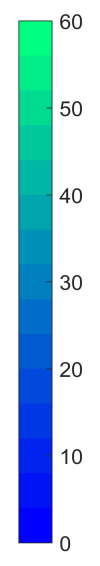

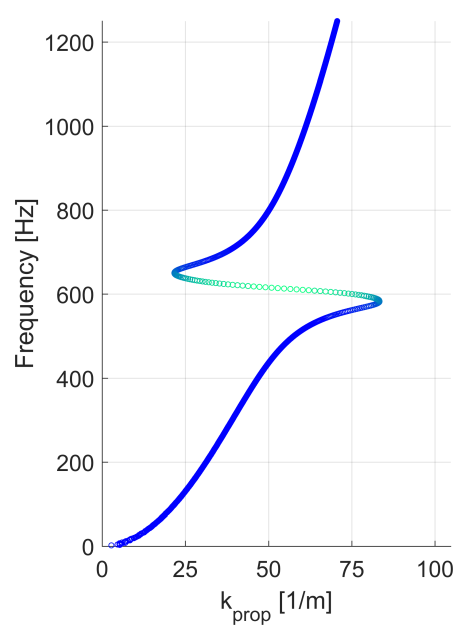

(a)

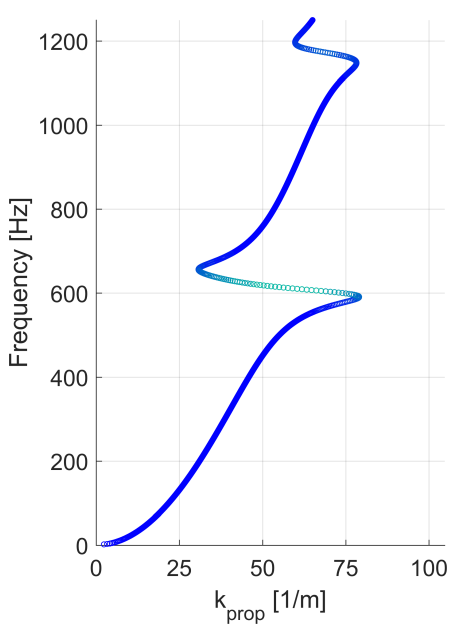

(b)

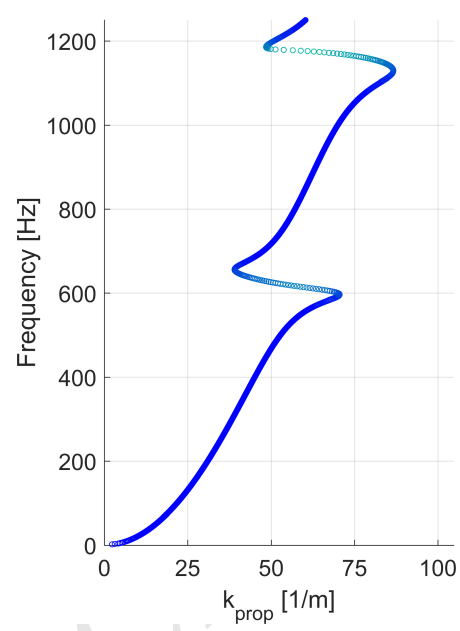

(c)

Figure 17 Dispersion curves obtained with the $\mu(\omega)$ approach for propagation directions $\theta=0^{\circ}$ (a), $45^{\circ}$ (b) and $90^{\circ}$ (c), coloured according to the attenuation, denoted by $|\operatorname{Im}(k)|$.

out-of-plane component which can lead to additional (un)desired resonant band gap behaviour for the flexural wave mode in case the subwavelength criterion is satisfied.

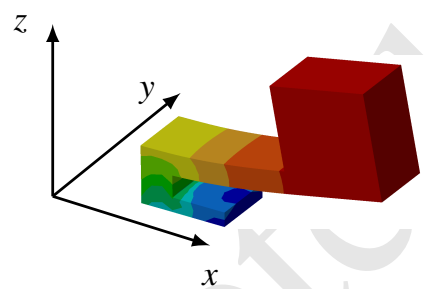

(a)

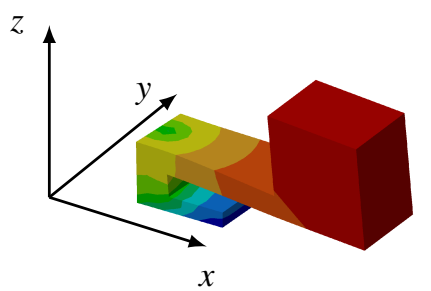

(b)

Figure 18 First mode at $616 \mathrm{~Hz}$ (a) and second mode at $1214 \mathrm{~Hz}$ (b) of the resonant structure with clamped base.

Around the designed stop band frequency range, the high damping in the resonator results in an evident looping back of the solutions. As observed in Sec. 3.3.1, the size of these complex solution loops varies with the propagation direction $\theta$. This leads to the variation of the eventual attenuation performance with $\theta$ as observed in Fig. 17, with $\theta=0^{\circ}$ showing the highest attenuation. The low damping in the host structure causes the regions away from the stop band to be largely unaffected, which leads to the very low predicted attenuation.

Apart from the designed stop band zone, the effect of the second resonator mode can be observed in the dispersion curves. For the considered UC dimensions, the second resonator mode still satisfies the subwavelength requirement around the resonance frequency at $1214 \mathrm{~Hz}$ in order to yield resonant band gap behaviour. The asymmetry of the 
second resonator mode, however, causes directional band gap behaviour, which does not lead to a stop band for the flexural wave mode along the entire IBC: an outspoken zone of attenuation is present around the second resonance frequency for the $\theta=90^{\circ}$ direction and to a lesser extent for the $\theta=45^{\circ}$ direction, while no attenuation zone is observed for the $\theta=0^{\circ}$ direction. These zones correspond to undamped band gaps between $1119-1171 \mathrm{~Hz}$ and 1161 - $1171 \mathrm{~Hz}$ for $\theta=90^{\circ}$ and $\theta=45^{\circ}$ respectively. This directional dependency is explained by the in-plane nature of this second mode, requiring an excitation component of the resonator basis in the $y$-direction for the flexural waves in the host structure to couple with the sideways swaying motion and thus the resulting out-of-plane exerted force by this resonator mode. The effect of damping in these second resonant band gap frequency regions corresponds to the earlier observations. Damping causes the directional band gaps to close gradually, forming broader zones of attenuation at the expense of reduced peak performance. While the $\theta=90^{\circ}$ direction shows similar attenuation to the first band gap frequency region, the $\theta=45^{\circ}$ directions the considerably narrower band gap leads to nearly closed dispersion curves.

For this type of beam shaped resonator with end point mass, the beam, mass and foot partitions can be modified geometrically or adapted towards multi-material assemblies. This way, resonance frequencies can be changed, multiple consecutive full or directional stop band regions can be created by exploiting consecutive resonator modes or the resonator modes could be spread such that full or directional stop band regions are obtained in specific consecutive frequency zones. The presented $\mu(\omega)$ unit cell analysis serves as a useful tool to assess the impact of these resonator modes and the influence of damping on the, possibly directional, attenuation performance. Damping effects can further be modified by selecting a material with higher/lower intrinsic damping for both host structure and resonator, depending on the frequency region of interest. Damped resonators could for instance serve as more efficient mass additions for attenuation in a confined frequency region, as compared to the addition of damping layers to the host structure. Further, additional damping treatments can be added to and/or in between resonator and host structure, the effects of which can be analysed using the presented $\mu(\omega)$ unit cell analysis.

\section{Experimental validation}

In this section, the damped dispersion curves obtained with the $\mu(\omega)$ UC approach are validated using measured dispersion curves. After introducing the EIWC method, the experimental setup is described. Next, the measured dispersion curves are presented and compared with the numerical predictions.

\subsection{Extended Inhomogeneous Wave Correlation}

The application of the $\mu(\omega)$ UC analysis approach, as introduced in Sec. 2, allows LRMs with damping to be analysed by means of dispersion curves with complex propagation constants $\mu$. These dispersion curves represent the damped plane wave propagation behaviour for infinite periodic structures. Infinite metamaterial structures can however not be manufactured. Hence, the real life performance of their finite size counterparts should be considered. As discussed in [55], the eigenfrequencies and modes of finite size LRM structures are obtained by sampling of the 
undamped dispersion curves of their infinite periodic counterparts. Consequently, as no free wave propagation is found in the stop band frequency zone, no eigenfrequencies for the finite size metamaterial are obtained in that zone for an undamped, finite metamaterial structure. In order to validate the obtained numerical predictions for damped LRMs, as presented in Sec. 3, measurements are performed on a finite size realisation to identify experimental dispersion curves for various propagation directions.

In this work, experimental dispersion curves are retrieved from harmonic out-of-plane displacement field measurements $w(x, y)$ of a vibrating planar structure by extending the IWC approach [44] with the experimental excitation location. The harmonic displacement field $w(x, y)$ is realised by a harmonic normal point force at a location $\left(x_{0}, y_{0}\right)$ on the metamaterial structure. The EIWC approach correlates this displacement field $w(x, y)$ with an inhomogeneous wave in order to extract dispersion curves. For the considered metamaterial structure, strong attenuation is expected around the band gap frequency zones. Since the objective is to verify the predicted complex propagation constant behaviour mainly in these regions, it is proposed here to define an inhomogeneous wave including the excitation location $\left(x_{0}, y_{0}\right)$ to obtain a better estimate of the attenuation. Following extended inhomogeneous wave $\hat{o}_{k_{\text {prop }}, k_{d e c}, \theta}$ is proposed:

$$
\hat{o}_{k_{\text {prop }}, k_{d e c}, \theta}(x, y)=e^{-\mathrm{i}\left(k_{\text {prop }}(\theta)+\mathrm{i} k_{\text {dec }}(\theta)\right)\left|\left(x-x_{0}\right) \cos (\theta)+\left(y-y_{0}\right) \sin (\theta)\right|},
$$

with $k_{\text {prop }}$ and $k_{d e c}$ the propagating and decaying part of the wavenumber $k=k_{\text {prop }}+\mathrm{i} k_{d e c}$ and $\theta$ the propagation direction in the $x y$-plane. This inhomogeneous wave is a spatially decaying plane wave, originating at the excitation location $\left(x_{0}, y_{0}\right)$, corresponding to the plane wave solutions arising from the UC analysis with damping for which the propagation constant $\mu$ and the wavenumber $k$ are related as in Eq. (13). The extended inhomogeneous wave is correlated with the measured wave field as follows [44]:

$$
\operatorname{EIWC}\left(k_{\text {prop }}, k_{d e c}, \theta\right)=\frac{\left|\sum_{i=1}^{N} w\left(x_{i}, y_{i}\right) \hat{\sigma}_{k_{p r o p}, k_{d e c}, \theta}^{*}\left(x_{i}, y_{i}\right) \rho\left(x_{i}, y_{i}\right) S_{i}\right|}{\sqrt{\sum_{i=1}^{N}\left|w\left(x_{i}, y_{i}\right)\right|^{2} \rho\left(x_{i}, y_{i}\right) S_{i} \sum_{i=1}^{N}\left|\hat{o}_{k_{p r o p}, k_{d e c}, \theta}\left(x_{i}, y_{i}\right)\right|^{2} \rho\left(x_{i}, y_{i}\right) S_{i}}},
$$

with * denoting the complex conjugate, $N$ the amount of measured points $\left(x_{i}, y_{i}\right), S_{i}$ an estimation of the surface around the $i$-th measurement point and $\rho$ the coherence of the measurement data to take the influence of measurement noise into account. In this work, a regular rectangular grid of $N_{x}$ and $N_{y}$ measurement points in $x$-and $y$-directions is used.

By maximisation of the $\operatorname{EIWC}\left(k_{\text {prop }}, k_{d e c}, \theta\right)$ at the considered frequencies of interest to $k_{\text {prop }}$ and $k_{d e c}$ for the given propagation directions $\theta$, experimental dispersion curves are retrieved [44, 56, 57], which can be compared to the numerical dispersion curves calculated using the $\mu(\omega)$ UC analysis approach.

\subsection{Description measurement setup}

For the validation of the numerical dispersion curves with damping, measurements are performed on a finite size metamaterial plate of the design introduced in Sec. 3.1. The metamaterial plate is manufactured by adding a total of 400 resonators to a $1 \mathrm{~mm}$ thick $60 \times 60 \mathrm{~cm}$ aluminium plate host structure (see Fig. 19b), leading to a grid of $20 \times 20$ unit cells centred on the plate, oriented with the $x$-direction as indicated in Fig. 19a. 


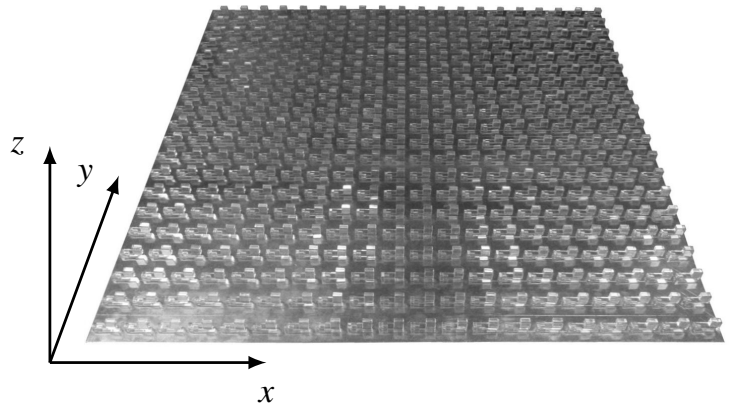

(a)

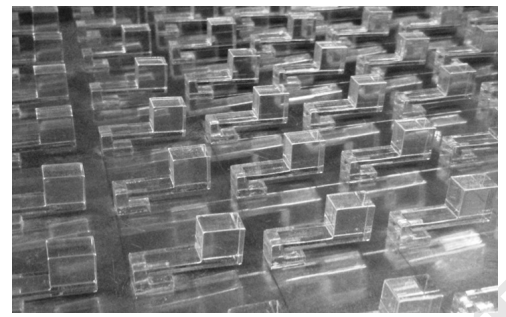

(b)

Figure 19 Manufactured locally resonant metamaterial plate (a) and close-up view (b).

SLDV measurements are performed using a Polytec PSV-500 Scanning Vibrometer [58] to obtain the harmonic structural displacement field $w(x, y)$ of the plate subjected to a shaker excitation as shown in Fig. 20. To avoid exciting the structure in a nodal position, the shaker is attached to the metamaterial plate through an impedance head at an asymmetric off-center location, at $(-5.6,-3.2) \mathrm{cm}$ from the plate center, with $x$ and $y$ conventions according to Fig. 19a. The $60 \times 60 \mathrm{~cm}$ metamaterial plate is suspended with two wires along the $x=60 \mathrm{~cm}$ side to mimic free boundary conditions. The out-of-plane structural displacement field $w(x, y)$ is measured as the receptance frequency response function between the input force measured at the impedance head and the displacement responses. A white noise excitation signal is used and the measurements are acquired from $10 \mathrm{~Hz}$ to $1250 \mathrm{~Hz}$ at 3200 frequency lines.

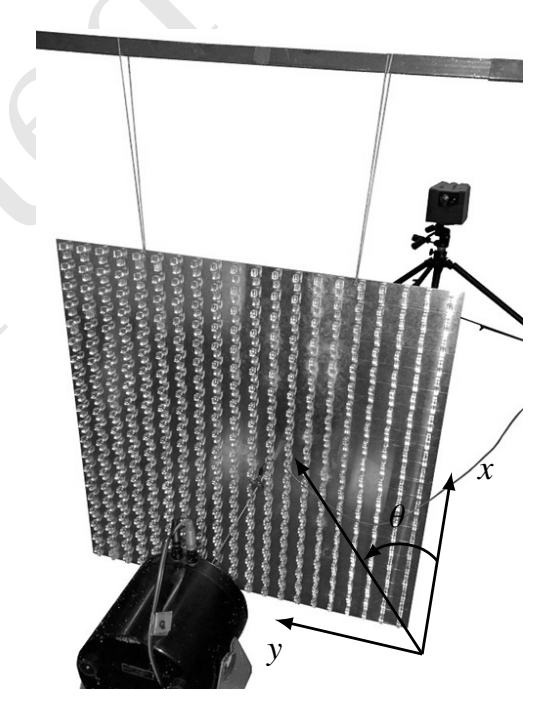

Figure 20 SLDV measurement setup with freely suspended metamaterial plate and electrodynamic shaker.

As indicated in Sec. 2, the wave vector content of interest is located inside the first Brillouin zone bounded by $\operatorname{Re}\left(\mu_{x}, \mu_{y}\right) \in[-\pi, \pi]$. These boundaries correspond to half wavelength points $\lambda_{x}=2 L_{x}$ and $\lambda_{y}=2 L_{y}$ for the $L_{x} \times L_{y}$ 
unit cell. Consequently, to satisfy the sampling theorem of the underlying relation with the Discrete Fourier Transform [44], the spacing of the grid should be $\Delta \leq \sqrt{L_{x}^{2}+L_{y}^{2}} / 2$. Although in the frequency range of interest the boundaries of the first Brillouin zone for the metamaterial with damping are no longer attained around the stop band frequency region, a spacing of $\Delta_{x}=L_{x} / 2=1.5 \mathrm{~cm}$ and $\Delta_{y}=L_{y} / 2=1.5 \mathrm{~cm}$ is adopted. The responses are hence obtained for an equidistant grid of $N_{x}=41$ by $N_{y}=41$ points located at each of the UC corners.

\subsection{Measured dispersion curves}

In a first step, the EIWC is calculated considering a purely propagating wave, by imposing the decaying part $k_{d e c}=0$ in Eq. (18), for the three propagation directions $\theta=0^{\circ}, 45^{\circ}$ and $90^{\circ}$. The EIWC results are presented in Fig. 21, with the colour scale indicating the EIWC magnitude. Since out-of-plane displacement field measurements are used, only the flexural wavenumber spectrum is retrieved.

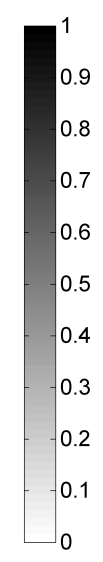

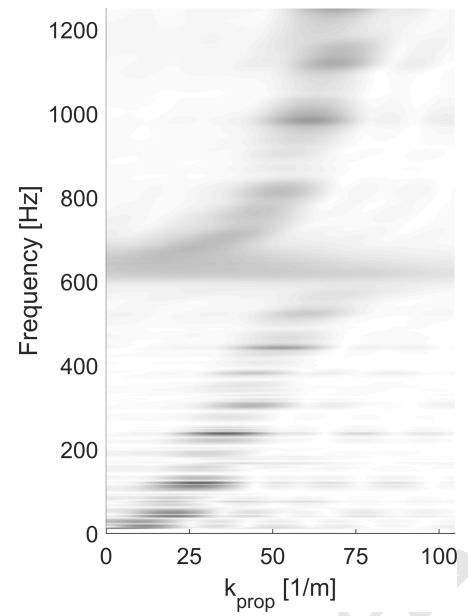

(a)

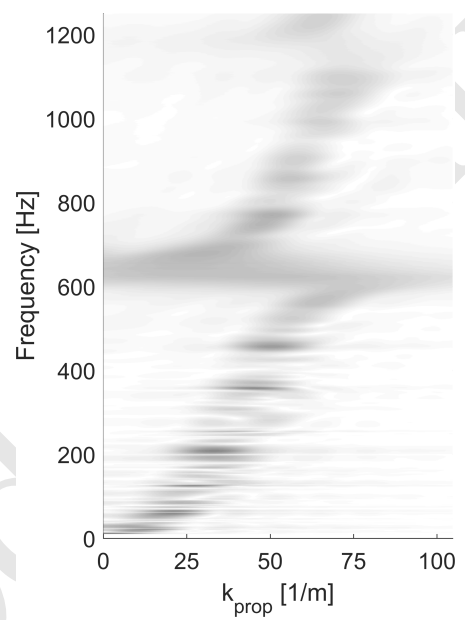

(b)

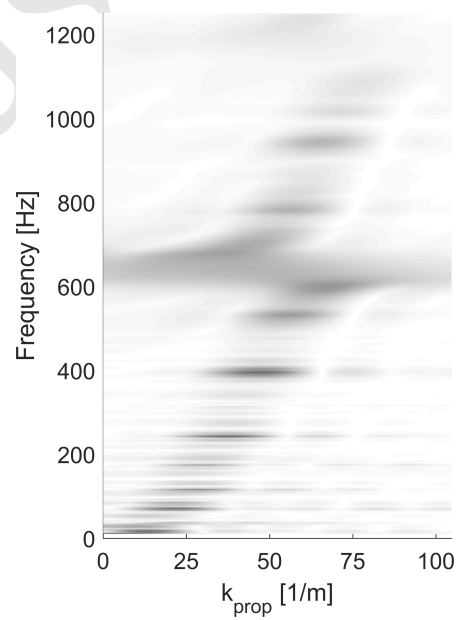

(c)

Figure 21 Calculated EIWC for different propagation directions $\theta=0^{\circ}$ (a), $45^{\circ}$ (b) and $90^{\circ}$ (c), considering no attenuation, coloured according to the EIWC magnitude.

In the lower frequency range, the structure is mainly dominated by modal behaviour. Corresponding to the findings in [55], these structural modes show up as EIWC points with a high magnitude, sampling on the dispersion curves. Around the designed stop band frequency range, no freely propagating waves are allowed in the structure and hence, no structural modes are observed in the EIWC results. Instead, zones of high dispersion are obtained, indicating the presence of stop band behaviour in the manufactured sample. The directionality of the stop band behaviour is reflected in the EIWC spectrum, as the high dispersion zone around the first band gap tightens towards $\theta=90^{\circ}$. For the directional band gap related to the second resonator mode, more outspoken differences are observed along the various propagation directions. The $\theta=90^{\circ}$ and $45^{\circ}$ directions show a clear discontinuity of the flexural mode dispersion curve, corresponding to the $\mathrm{UC}$ analysis results, while for the $\theta=0^{\circ}$ direction no such gap is present. 
In order to obtain dispersion curves for the propagation directions of interest, the EIWC is maximised with respect to $k_{\text {prop }}$ and $k_{d e c}$ for each frequency. The results are presented in Fig. 22; the colour scale again indicates the magnitude of the EIWC values. Clear dispersion curves are extracted for the three propagation directions. As observed for the propagating wavenumber spectrum, in the modally dominated lower frequency range, modes appear as discrete points of high EIWC magnitude connected by lower EIWC magnitude regions. In the higher frequency range, these rather discrete high EIWC magnitude points are smeared out due to the damping present in the structure. Around the designed stop band frequency range, the extracted dispersion curves bend back and show a closing of the flexural wavemode branches, corresponding to the complex $\mu$ solution loops predicted by the $\mu(\omega)$ UC approach. The directional dependency is captured as the frequency range and propagating wavenumber range of the transition decreases when varying the propagation direction from $\theta=0^{\circ}$ to $\theta=90^{\circ}$. Also around the second band gap, the directional band gap behaviour is reflected well in the measurements. Along the $\theta=0^{\circ}$ direction no band gap is found, while the $\theta=45^{\circ}$ and $\theta=90^{\circ}$ directions show the presence of band gap behaviour, with the transition of the latter covering a larger frequency and propagating wavenumber range.

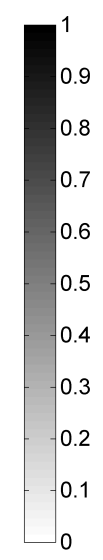

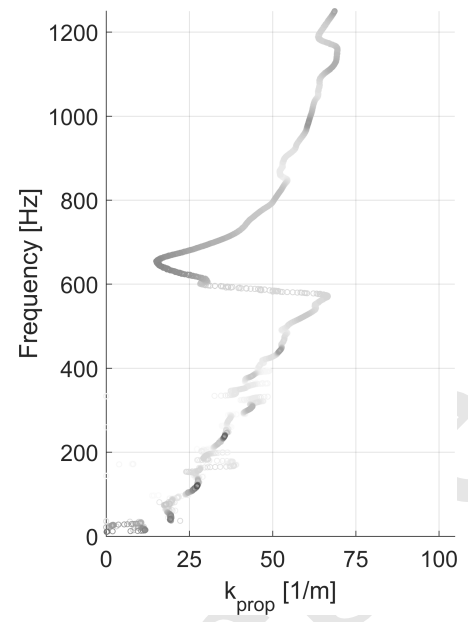

(a)

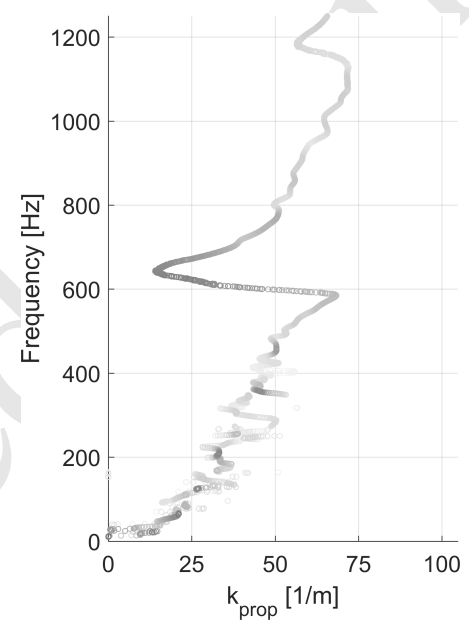

(b)

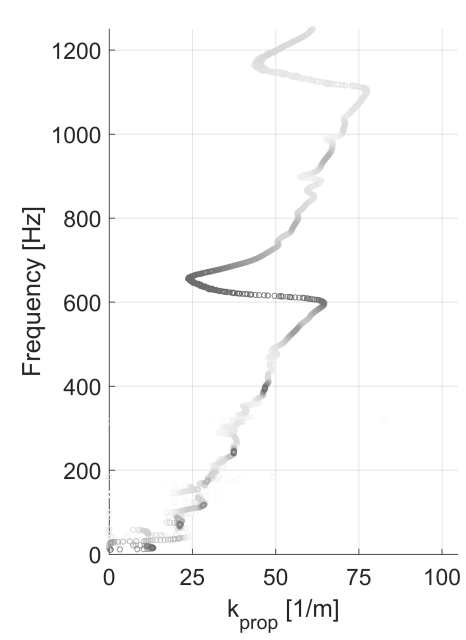

(c)

Figure 22 Measured dispersion curves obtained by maximising the EIWC for different propagation directions $\theta=0^{\circ}$ (a), $45^{\circ}$ (b) and $90^{\circ}$ (c), coloured according to the EIWC magnitude.

Above observations become more clear when comparing the experimentally obtained dispersion curves to the predictions from the damped UC modelling approach. In Fig. 23, the propagating wavenumber is compared by displaying the measured dispersion curves together with the UC results, coloured in black, while Fig. 24 shows the propagating part of the measured wavenumber, coloured according to the magnitude of the decaying part of the wavenumber $|\operatorname{Im}(k)|=\left|k_{d e c}\right|$. For all three propagation directions a very good agreement is found over the considered frequency range, both around as well as inside the stop band zones. In Fig. 23 below and in between the stop band 
frequency ranges, the modes clearly sample on the dispersion curves. Some discrepancies are observed mainly at lower frequencies, which can be related to the free-free boundary conditions for which the modeshapes differ from the sinusoidal wave shapes in the infinite periodic structure. The presence of damping reduces this influence with increasing frequency. Furthermore, the mass loading of the shaker excitation can be of influence. For the attenuation in Fig. 24, outside the stop band zones, very good agreement is found with the numerically predictions in Fig. 17 and only in between modes, a slight increase of the attenuation is observed. This indicates that no particular attenuation occurs in the metamaterial sample in these frequency ranges.

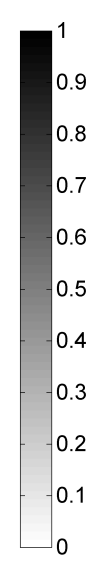

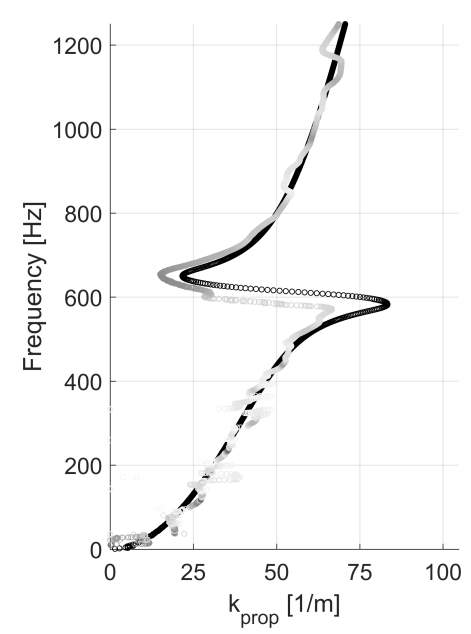

(a)

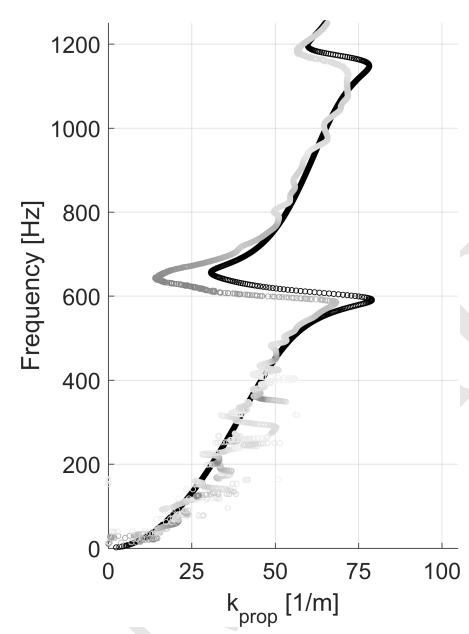

(b)

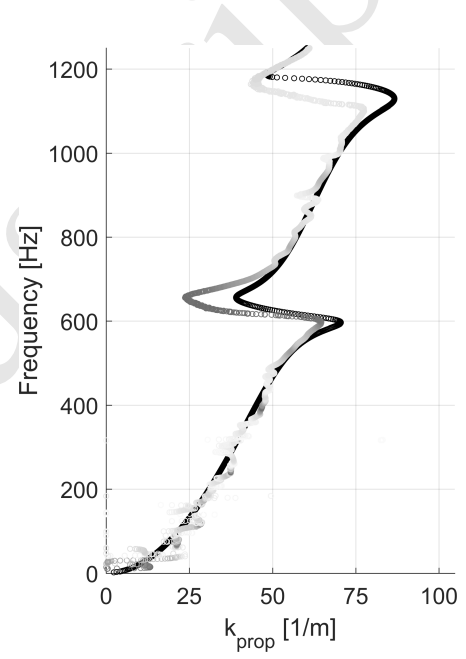

(c)

Figure 23 Comparison of the measured dispersion curves from Fig. 22, coloured according to the EIWC magnitude, with the numerically predicted damped dispersion curves from Fig. 17, coloured in black, for different propagation directions $\theta=0^{\circ}$ (a), $45^{\circ}$ (b) and $90^{\circ}$ (c).

In the first stop band frequency range, the complex loop transition is found in the manufactured sample, showing good correspondence with the predicted behaviour. Clear zones of increased attenuation are found, with the covered frequency and wavenumber ranges agreeing well for the three propagation directions. Where the UC predictions indicate decreasing complex loop sizes and attenuation performance when varying the propagation direction from $\theta=0^{\circ}$ to $\theta=90^{\circ}$, however, the measurements pick up similar attenuation for the three directions. Inspecting the second stop band zone, very good agreement with the predicted behaviour is found. The predicted more outspoken directional dependency is clearly reflected in the measured dispersion curves. Complex transition loops of increasing size are found for the $\theta=45^{\circ}$ and $\theta=90^{\circ}$ directions, while for $\theta=0^{\circ}$ only an effect is found comparable to the result in between modes, indicating no outspoken stop band behaviour. Comparing the measured attenuation for this frequency range in Fig. 24 with the predictions of Fig. 17, very good agreement is obtained.

Apart from the influence of the finite sample size in combination with the boundary conditions, discrepancies 


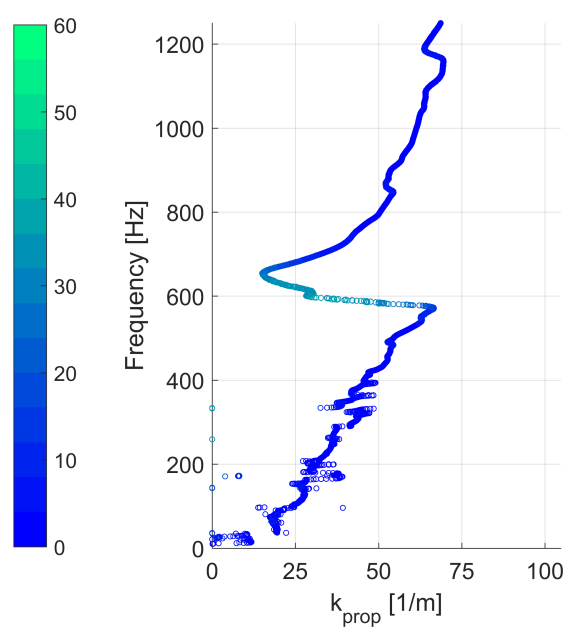

(a)

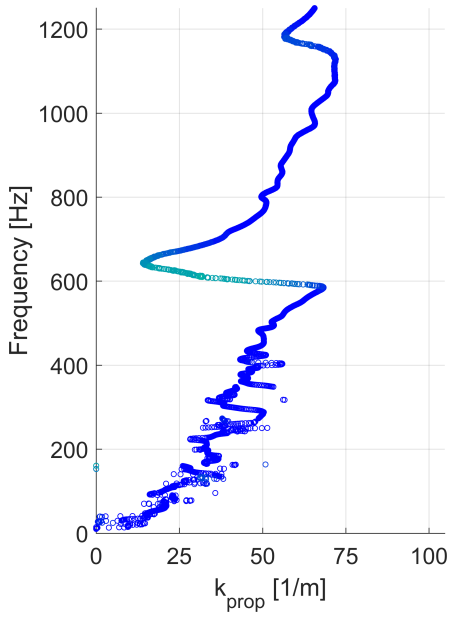

(b)

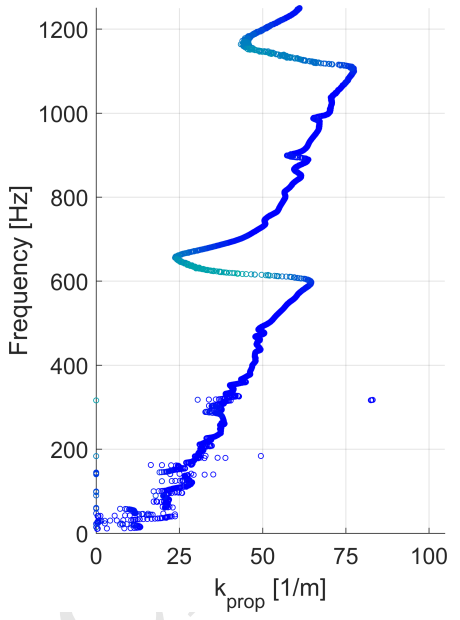

(c)

Figure 24 Measured dispersion curves, coloured according to $|\operatorname{Im}(k)|=\left|k_{d e c}\right|$, for different propagation directions $\theta=0^{\circ}$ (a), $45^{\circ}$ (b) and $90^{\circ}$ (c).

between measurements and predictions can arise from the fact that both the EIWC and UC analysis assume plane wave propagation, while the shaker excitation leads to a circular wave pattern. Also the scatter of the periodicity, resonator dimensions, alignment and adhesive thickness can induce similar, albeit less outspoken broadening and peak performance reducing effects [59], but can also cause the predicted strictly directional dependency to be less prominent in the measurements. It is in addition noted that differences can originate from the basic damping model used in the numerical model with constant damping loss factors obtained through measurements.

Overall, very good agreement is found between the experimental results and numerical predictions for various propagation directions. These results demonstrate the predictive value of the $\mu(\omega)$ UC modelling approach for LRMs with damping, able to capture the trends and attenuation performance for the complex $\mu$ transition loops around the stop band frequency zones, and confirm the importance of predicting the wave propagation behaviour for damped structures not merely along the irreducible Brillouin contour, but throughout the irreducible Brillouin zone.

\section{Conclusion}

This paper applies a $\mu(\omega)$ unit cell analysis approach including damping to analyse dispersion curves for the time harmonic response of locally resonant metamaterials, by solving a polynomial eigenvalue problem for complex propagation constants, denoting spatial decay, at given real frequencies and propagation directions. A realisable locally resonant metamaterial design with flexural stop band behaviour is introduced, consisting of a plate host structure with beam shaped resonators, and the wave solutions in and around the stop band frequency range are compared with the commonly used $\omega(\mu)$ unit cell method. Excellent agreement is found between the $\mu(\omega)$ and $\omega(\mu)$ approach for an 
undamped unit cell model. The effect of damping in the locally resonant metamaterial is investigated by calculating the complex dispersion curves along various propagation directions and representing them in the complex propagation constant space for a unit cell containing damping in only the resonator, only the host structure and both in the resonator and host structure. Addition of damping leads to complex propagation constants and is found to cause the dispersion curves before and after the stop band to close by means of a loop of complex propagation constant solutions. By increasing the damping, the decaying part of these complex solutions reduces, while the frequency range they cover increases, leading to a broader frequency range of attenuation at the expense of peak attenuation performance. While damping in the resonators affects mainly the frequency range in and around the stop band, damping in the host structure mainly affects the zone outside the stop band region. The effect of damping in the resonators is found to be considerably more important than the host structure, for the considered locally resonant metamaterial design. The numerical dispersion curves calculated with the $\mu(\omega)$ unit cell approach are validated by experimental measurements of dispersion curves on a manufactured metamaterial sample, based on the Extended Inhomogeneous Wave Correlation method. The numerical predictions are in very good agreement with the measurements, demonstrating the predictive value of the presented $\mu(\omega)$ unit cell modelling approach for the analysis of damped metamaterial structures.

\section{Acknowledgements}

The Research Foundation - Flanders (F.W.O.) is gratefully acknowledged for its support. The research of L. Van Belle is funded by a doctoral grant from the Research Foundation - Flanders (11ZH817N). E. Deckers is a postdoctoral fellow of the Research Foundation - Flanders (12D2614N). The authors gratefully acknowledge the KU Leuven Research Fund for their support through an IOF-Leverage project.

\section{Appendix A. Coefficient matrices of the polynomial eigenvalue problem}

The coefficient matrices $\tilde{\mathbf{D}}_{i}$ of the eigenvalue problem in Eq. (11) are elaborated below.

$$
\begin{gathered}
\tilde{\mathbf{D}}_{1}=\left[\begin{array}{ccc}
\mathbf{Q}_{B L, T R} \mathbf{D}_{T R, B L} & \mathbf{Q}_{B L, T R} \mathbf{D}_{T R, L} & \mathbf{Q}_{B L, T R} \mathbf{D}_{T R, B} \\
\mathbf{0} & \mathbf{0} & \mathbf{0} \\
\mathbf{0} & \mathbf{0} & \mathbf{0}
\end{array}\right] \\
\tilde{\mathbf{D}}_{2}=\left[\begin{array}{ccc}
\mathbf{Q}_{B L, T L} \mathbf{D}_{T L, B L}+\mathbf{Q}_{B L, T R} \mathbf{D}_{T R, B R} \mathbf{Q}_{B R, B L} & \mathbf{Q}_{B L, T L} \mathbf{D}_{T L, L}+\mathbf{Q}_{B L, T R} \mathbf{D}_{T R, R} \mathbf{Q}_{R, L} & \mathbf{Q}_{B L, T L} \mathbf{D}_{T L, B} \\
\mathbf{0} & \mathbf{0} & \mathbf{0} \\
\mathbf{Q}_{B, T} \mathbf{D}_{T, B L} & \mathbf{Q}_{B, T} \mathbf{D}_{T, L} & \mathbf{Q}_{B, T} \mathbf{D}_{T, B}
\end{array}\right] \\
\tilde{\mathbf{D}}_{3}=\left[\begin{array}{ccc}
\mathbf{Q}_{B L, B R} \mathbf{D}_{B R, B L}+\mathbf{Q}_{B L, T R} \mathbf{D}_{T R, T L} \mathbf{Q}_{T L, B L} & \mathbf{Q}_{B L, B R} \mathbf{D}_{B R, L} & \mathbf{Q}_{B L, B R} \mathbf{D}_{B R, B}+\mathbf{Q}_{B L, T R} \mathbf{D}_{T R, T} \mathbf{Q}_{T, B} \\
\mathbf{Q}_{L, R} \mathbf{D}_{R, B L} & \mathbf{Q}_{L, R} \mathbf{D}_{R, L} & \mathbf{Q}_{L, R} \mathbf{D}_{R, B} \\
\mathbf{0} & \mathbf{0} & \mathbf{0}
\end{array}\right]
\end{gathered}
$$




$$
\begin{aligned}
& \tilde{\mathbf{D}}_{4}=\left[\begin{array}{c}
\mathbf{D}_{B L, B L}+\mathbf{Q}_{B L, B R} \mathbf{D}_{B R, B R} \mathbf{Q}_{B R, B L}+\mathbf{Q}_{B L, T L} \mathbf{D}_{T L, T L} \mathbf{Q}_{T L, B L}+\mathbf{Q}_{B L, T R} \mathbf{D}_{T R, T R} \mathbf{Q}_{T R, B L} \\
\mathbf{D}_{L, B L}+\mathbf{Q}_{L, R} \mathbf{D}_{R, B R} \mathbf{Q}_{B R, B L} \\
\mathbf{D}_{B, B L}+\mathbf{Q}_{B, T} \mathbf{D}_{T, T L} \mathbf{Q}_{T L, B L}
\end{array} \ldots\right. \\
& \left.\mathbf{D}_{B L, L}+\mathbf{Q}_{B L, B R} \mathbf{D}_{B R, R} \mathbf{Q}_{R, L} \quad \mathbf{D}_{B L, B}+\mathbf{Q}_{B L, T L} \mathbf{D}_{T L, T} \mathbf{Q}_{T, B}\right] \\
& \begin{array}{lll}
\ldots & \mathbf{D}_{L, L}+\mathbf{Q}_{L, R} \mathbf{D}_{R, R} \mathbf{Q}_{R, L} \quad \mathbf{D}_{L, B}
\end{array} \\
& \mathbf{D}_{B, L} \quad \mathbf{D}_{B, B}+\mathbf{Q}_{B, T} \mathbf{D}_{T, T} \mathbf{Q}_{T, B} \\
& \tilde{\mathbf{D}}_{5}=\left[\begin{array}{ccc}
\mathbf{Q}_{B L, T L} \mathbf{D}_{T L, B R} \mathbf{Q}_{B R, B L} & \mathbf{Q}_{B L, T L} \mathbf{D}_{T L, R} \mathbf{Q}_{R, L} & \mathbf{0} \\
\mathbf{0} & \mathbf{0} & \mathbf{0} \\
\mathbf{Q}_{B, T} \mathbf{D}_{T, B R} \mathbf{Q}_{B R, B L} & \mathbf{Q}_{B, T} \mathbf{D}_{T, R} \mathbf{Q}_{R, L} & \mathbf{0}
\end{array}\right] \\
& \tilde{\mathbf{D}}_{6}=\left[\begin{array}{ccc}
\mathbf{Q}_{B L, B R} \mathbf{D}_{B R, T L} \mathbf{Q}_{T L, B L} & \mathbf{0} & \mathbf{Q}_{B L, B R} \mathbf{D}_{B R, T} \mathbf{Q}_{T, B} \\
\mathbf{Q}_{L, R} \mathbf{D}_{R, T L} \mathbf{Q}_{T L, B L} & \mathbf{0} & \mathbf{Q}_{L, R} \mathbf{D}_{R, T} \mathbf{Q}_{T, B} \\
\mathbf{0} & \mathbf{0} & \mathbf{0}
\end{array}\right] \\
& \tilde{\mathbf{D}}_{7}=\left[\begin{array}{ccc}
\mathbf{D}_{B L, B R} \mathbf{Q}_{B R, B L}+\mathbf{Q}_{B L, T L} \mathbf{D}_{T L, T R} \mathbf{Q}_{T R, B L} & \mathbf{D}_{B L, R} \mathbf{Q}_{R, L} & \mathbf{0} \\
\mathbf{D}_{L, B R} \mathbf{Q}_{B R, B L} & \mathbf{D}_{L, R} \mathbf{Q}_{R, L} & \mathbf{0} \\
\mathbf{D}_{B, B R} \mathbf{Q}_{B R, B L}+\mathbf{Q}_{B, T} \mathbf{D}_{T, T R} \mathbf{Q}_{T R, B L} & \mathbf{D}_{B, R} \mathbf{Q}_{R, L} & \mathbf{0}
\end{array}\right] \\
& \tilde{\mathbf{D}}_{8}=\left[\begin{array}{ccc}
\mathbf{D}_{B L, T L} \mathbf{Q}_{T L, B L}+\mathbf{Q}_{B L, B R} \mathbf{D}_{B R, T R} \mathbf{Q}_{T R, B L} & \mathbf{0} & \mathbf{D}_{B L, T} \mathbf{Q}_{T, B} \\
\mathbf{D}_{L, T L} \mathbf{Q}_{T L, B L}+\mathbf{Q}_{L, R} \mathbf{D}_{R, T R} \mathbf{Q}_{T R, B L} & \mathbf{0} & \mathbf{D}_{L, T} \mathbf{Q}_{T, B} \\
\mathbf{D}_{B, T L} \mathbf{Q}_{T L, B L} & \mathbf{0} & \mathbf{D}_{B, T} \mathbf{Q}_{T, B}
\end{array}\right]
\end{aligned}
$$

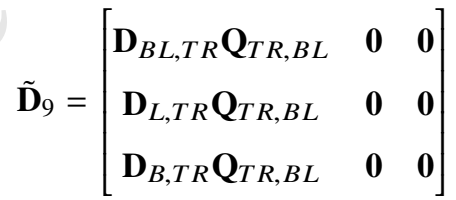

\section{References}

[1] A. I. Taub, P. E. Krajewski, A. A. Luo, and J. N. Owens. The evolution of technology for materials processing over the last 50 years: The automotive example. JOM, 59(2):48-57, 2007.

[2] M. Basner, W. Babisch, A. Davis, M. Brink, C. Clark, S. Janssen, and S. Stansfeld. Auditory and non-auditory effects of noise on health. The Lancet, 383(9925):1325-1332, 2014.

[3] L. Brillouin. Wave propagation in periodic structures. McGraw-Hill Book Company, 2nd edition, 1946.

[4] M.-H. Lu, L. Feng, and Y.-F. Chen. Phononic crystals and acoustic metamaterials. Materials Today, 12(12):34-42, 2009.

[5] P. A. Deymier. Acoustic metamaterials and phononic crystals, volume 173. Springer, 2013.

[6] J. O. Vasseur, P. A. Deymier, G. Frantziskonis, G. Hong, B. Djafari-Rouhani, and L. Dobrzynski. Experimental evidence for the existence of absolute acoustic band gaps in two-dimensional periodic composite media. Journal of Physics: Condensed Matter, 10(27):6051, 1998. 
[7] J. O. Vasseur, P. A. Deymier, B. Djafari-Rouhani, Y. Pennec, and A.-C. Hladky-Hennion. Absolute forbidden bands and waveguiding in two-dimensional phononic crystal plates. Physical Review B, 77(8):085415, 2008.

[8] Z. Liu, C. T. Chan, P. Sheng, A. L. Goertzen, and J. H. Page. Elastic wave scattering by periodic structures of spherical objects: Theory and experiment. Physical Review B, 62(4):2446, 2000.

[9] Z. Liu, X. Zhang, Y. Mao, Y. Y. Zhu, Z. Yang, C. T. Chan, and P. Sheng. Locally resonant sonic materials. Science, 289(5485):1734-1736, Sep 2000.

[10] P. Sheng, X. X. Zhang, Z. Liu, and C. T. Chan. Locally resonant sonic materials. Physica B: Condensed Matter, 338(1):201-205, 2003.

[11] X. Zhou, X. Liu, and G. Hu. Elastic metamaterials with local resonances: An overview. Theoretical and Applied Mechanics Letters, 2(4):041001, 2012.

[12] C. Goffaux, J. Sánchez-Dehesa, A. L. Yeyati, P. Lambin, A. Khelif, J. O. Vasseur, and B. Djafari-Rouhani. Evidence of Fano-like interference phenomena in locally resonant materials. Physical Review Letters, 88(22), May 2002.

[13] C. Claeys, K. Vergote, P. Sas, and W. Desmet. On the potential of tuned resonators to obtain low-frequency vibrational stop bands in periodic panels. Journal of Sound and Vibration, 332(6):1418-1436, Mar 2013.

[14] S. Yao, X. Zhou, and G. Hu. Experimental study on negative effective mass in a 1d mass-spring system. New Journal of Physics, 10(4):043020, 2008.

[15] L. Airoldi and M. Ruzzene. Design of tunable acoustic metamaterials through periodic arrays of resonant shunted piezos. New Journal of Physics, 13(11):113010, 2011.

[16] M. Oudich, M. Senesi, M. B. Assouar, M. Ruzenne, J.-H. Sun, B. Vincent, Z. Hou, and T.-T. Wu. Experimental evidence of locally resonant sonic band gap in two-dimensional phononic stubbed plates. Physical Review B, 84(16), Oct 2011.

[17] C. Claeys, M. Vivolo, P. Sas, and W. Desmet. Study of honeycomb panels with local cell resonators to obtain low-frequency vibrational stopbands. In Proceedings of DYNACOMP 2012, Arcachon, France, 22-24 May 2012. art.nr. AA - DYNACOMP - 2012 - 20.

[18] Y. Xiao, J. Wen, L. Huang, and X. Wen. Analysis and experimental realization of locally resonant phononic plates carrying a periodic array of beam-like resonators. Journal of Physics D: Applied Physics, 47(4):045307, 2014.

[19] C. Sugino, S. Leadenham, M. Ruzzene, and A. Erturk. On the mechanism of bandgap formation in locally resonant finite elastic metamaterials. Journal of Applied Physics, 120(13):134501, oct 2016.

[20] C. Claeys, N. G. R. de Melo Filho, L. Van Belle, E. Deckers, and W. Desmet. Design and validation of metamaterials for multiple structural stop bands in waveguides. Extreme Mechanics Letters, Aug 2016. doi:10.1016/j.eml.2016.08.005.

[21] M. I. Hussein and M. J. Frazier. Metadamping: An emergent phenomenon in dissipative metamaterials. Journal of Sound and Vibration, 332(20):4767-4774, Sep 2013.

[22] J. M. Manimala and C. T. Sun. Microstructural design studies for locally dissipative acoustic metamaterials. Journal of Applied Physics, 115(2):023518, 2014.

[23] Y. Y. Chen, M. V. Barnhart, J. K. Chen, G. K. Hu, C. T.Sun, and G. L. Huang. Dissipative elastic metamaterials for broadband wave mitigation at subwavelength scale. Composite Structures, 136:358-371, Feb 2016.

[24] M. J. Frazier and M. I. Hussein. Generalized Bloch's theorem for viscous metamaterials: Dispersion and effective properties based on frequencies and wavenumbers that are simultaneously complex. C. R. Physique, 17:565-577, 2016.

[25] M. Collet, M. Ouisse, M. Ruzzene, and M. N. Ichchou. Floquet-Bloch decomposition for the computation of dispersion of two-dimensional periodic, damped mechanical systems. International Journal of Solids and Structures, 48(20):2837-2848, Oct 2011.

[26] M. Chen, D. Meng, H. Zhang, H. Jiang, and Y. Wang. Resonance-coupling effect on broad band gap formation in locally resonant sonic metamaterials. Wave Motion, 63:111-119, 2016.

[27] A. O. Krushynska, V. G. Kouznetsova, and M. G. D. Geers. Visco-elastic effects on wave dispersion in three-phase acoustic metamaterials. Journal of the Mechanics and Physics of Solids, Jul 2016.

[28] H. Peng, P. Frank Pai, and H. Deng. Acoustic multi-stopband metamaterial plates design for broadband elastic wave absorption and vibration suppression. International Journal of Mechanical Sciences, 103:104-114, Nov 2015. 
[29] D. Chronopoulos, I. Antoniadis, M. Collet, and M. Ichchou. Enhancement of wave damping within metamaterials having embedded negative stiffness inclusions. Wave Motion, May 2015.

[30] D. J. Mead. Wave propagation in continuous periodic structures: Research contributions from southampton, 1964-1995. Journal of Sound and Vibration, 190(3):495-524, 1996.

[31] M. I. Hussein, M. J. Leamy, and M. Ruzzene. Dynamics of phononic materials and structures: Historical origins, recent progress, and future outlook. Appl. Mech. Rev., 66(4):040802, May 2014.

[32] E. Andreassen and J. S. Jensen. Analysis of phononic bandgap structures with dissipation. Journal of Vibration and Acoustics, 135(4):041015, Jun 2013.

[33] A. R. Diaz, A. G. Haddow, and L. Ma. Design of band-gap grid structures. Struct Multidisc Optim, 29(6):418-431, Jan 2005.

[34] M. I. Hussein. Theory of damped Bloch waves in elastic media. Phys. Rev. B, 80(21), Dec 2009.

[35] M. I. Hussein and M. J. Frazier. Band structure of phononic crystals with general damping. Journal of Applied Physics, 108(9):093506, 2010.

[36] M. J. Frazier and M. I. Hussein. Viscous-to-viscoelastic transition in phononic crystal and metamaterial band structures. The Journal of the Acoustical Society of America, 138(5):3169-3180, 2015.

[37] F. Farzbod and M. J. Leamy. Analysis of Bloch's method in structures with energy dissipation. Journal of Vibration and Acoustics, 133(5):051010, 2011

[38] R. P. Moiseyenko and V. Laude. Material loss influence on the complex band structure and group velocity in phononic crystals. Phys. Rev. B, 83(6), Feb 2011.

[39] E. Manconi and B. R. Mace. Estimation of the loss factor of viscoelastic laminated panels from finite element analysis. Journal of Sound and Vibration, 329(19):3928-3939, Sep 2010

[40] B. R. Mace. Discussion of "Dynamics of phononic materials and structures: Historical origins, recent progress and future outlook" (M. I. Hussein, M. J. Leamy and M. Ruzzene, 2014, ASME Appl. Mech. Rev., 66(4), p. 040802). Appl. Mech. Rev., 66(4):045502, Jun 2014.

[41] B. R. Mace and E. Manconi. Modelling wave propagation in two-dimensional structures using finite element analysis. Journal of Sound and Vibration, 318(4-5):884-902, Dec 2008.

[42] B. Roozen, B. Verstraeten, L. Labelle, C. Glorieux, and P. Leclaire. Advanced dispersion measurement techniques for the characterization of the mechanical properties of poro-visco-elastic materials. In Proceedings of Internoise 2013, Innsbruck, Austria, 2013.

[43] F. Casadei, T. Delpero, A. Bergamini, P. Ermanni, and M. Ruzzene. Piezoelectric resonator arrays for tunable acoustic waveguides and metamaterials. Journal of Applied Physics, 112(6):064902, 2012.

[44] J. Berthaut, M. N. Ichchou, and L. Jezequel. K-space identification of apparent structural behaviour. Journal of Sound and Vibration, 280(3-5):1125-1131, Feb 2005.

[45] I. A. Veres, T. Berer, and O. Matsuda. Complex band structures of two dimensional phononic crystals: Analysis by the finite element method. Journal of Applied Physics, 114(8):083519, 2013.

[46] A. Palermo and A. Marzani. Extended bloch mode synthesis: Ultrafast method for the computation of complex band structures in phononic media. International Journal of Solids and Structures, 100:29-40, 2016.

[47] G. Wang, X. Wen, J. Wen, L. Shao, and Y. Liu. Two-dimensional locally resonant phononic crystals with binary structures. Physical Review Letters, 93(15), Oct 2004.

[48] L. Cremer, M. Heckl, and B. A. T. Petersson. Structure-borne sound: Structural Vibrations and Sound Radiation at Audio Frequencies. Springer Science \& Business Media, 3rd edition, 2005.

[49] W. Heylen, S. Lemmens, and P. Sas. Modal analysis theory and testing. KU Leuven, PMA Division, 2014.

[50] MSC Software. MSC Nastran 2016 reference manual, 2016.

[51] G. Radoičić and M. Jovanović. Experimental identification of overall structural damping of system. Strojniški vestnik-Journal of Mechanical Engineering, 59(4):260-268, 2013.

[52] M. Carfagni, E. Lenzi, and M. Pierini. The loss factor as a measure of mechanical damping. In Proceedings of the 16th International Modal Analysis Conference, 1998, volume 3243, pages 580-584, 1998. 
[53] V. Laude, Y. Achaoui, S. Benchabane, and A. Khelif. Evanescent Bloch waves and the complex band structure of phononic crystals. Phys. Rev. B, 80(9):092301, Sep 2009.

[54] J. G. McDaniel, P. Dupont, and L. Salvino. A wave approach to estimating frequency-dependent damping under transient loading. Journal of Sound and Vibration, 231(2):433-449, Mar 2000.

[55] C. Claeys, P. Sas, and W. Desmet. On the acoustic radiation efficiency of local resonance based stop band materials. Journal of Sound and Vibration, 333(14):3203-3213, Jul 2014.

[56] M. N. Ichchou, O. Bareille, and J. Berthaut. Identification of effective sandwich structural properties via an inverse wave approach. Engineering Structures, 30(10):2591-2604, Oct 2008.

[57] R. Cherif, J. D. Chazot, and N. Atalla. Damping loss factor estimation of two-dimensional orthotropic structures from a displacement field measurement. Journal of Sound and Vibration, 356:61-71, Nov 2015.

[58] Polytec GmbH. PSV-500 Scanning Vibrometer Datasheet. http://www.polytec.com/us/products/vibration-sensors/ scanning-vibrometers/psv-500-scanning-vibrometer/, 2016.

[59] C. Claeys. Design and Analysis of Resonant Metamaterials for Acoustic Insulation (Ontwerp en analyse van resonante metamaterialen voor geluidsisolatie). PhD thesis, KU Leuven - Faculty of Engineering Science, Arenberg Doctoral School, Apr 2014. 